\title{
OBSERVATION AND SYNTHESIS OF SPATIALLY-INCOHERENTWEAK-MOTION WAVEFIELDS AT ALFREDTON BASIN, NEW ZEALAND
}

\author{
A J Haines ${ }^{1}$ Jaishun Yu ${ }^{2}$
}

\begin{abstract}
SUMMARY
To observe and model the detailed pattern of ground motion amplification in a small soft-soil basin an experiment was conducted at Alfredton, New Zealand. 19 seismometers were deployed for 5 weeks at closely spaced sites in and around a 400-500 m diameter, sediment-filled depression in soft, sandstone basement. During this period 112 earthquakes, with "weak" ground motions, were detected by at least some of the instruments, and 15 well-recorded events were selected for detailed analysis. Geotechnical data obtained to provide the parameters for the 3-dimensional modelling included measurements of the shear-wave velocity. Across the basin this is $60 \mathrm{~m} / \mathrm{s}$ at the surface, increasing steadily to $300+\mathrm{m} / \mathrm{s}$ at the bottom of the basin, and the shear-wave velocity in the basement is $850 \mathrm{~m} / \mathrm{s}$. Thus, there are no boundaries where the contrast in shear-wave impedance is especially large
\end{abstract}

In contrast to situations where there are large contrasts in shear-wave impedance to trap seismic energy in soft-soil layers, the amplifications observed in the basin at Alfredton were small. The small amplifications are confirmed by the 3-dimensional modelling. Another feature of the observed wavefields is that in all cases the incident motions, recorded at the basement sites around the basin, were spatially incoherent. In other words, the wavefields arriving at the basin were of a complex, seemingly random nature. This is the first occasion that the spatial coherency of wavefields has been measured in a fine-scale experiment in New Zealand. Apart from the small amplications and the observed lack of coherency between the basement sites, the most striking result, which was obtained from both the observations and the modelling of similarly incoherent wavefields, is that for short-duration events in which the main motions last for no more than a second, the amplifications in the basin are larger than for events in which the motions are of longer duration; that is, the extent to which differently propagating incoherent wave packets interfere destructively inside the basin increases with the duration of the wavefields.

\section{INTRODUCTION}

In this paper we present observations and modelling results from an experiment at a small basin near Alfredton, northern Wairarapa, New Zealand. 19 identical portable seismographs were deployed in and around the basin for 5 weeks in 1993, during which 112 earthquakes were recorded, ranging in size from less than magnitude 2 to magnitude 5 . The purpose of the experiment was to identify the detailed pattern of ground motion amplification in the basin, as a guide to what can be expected in similar basins, and a secondary goal was to verify a recently developed package for modelling 3-dimensional seismic wave amplification in complex near-surface structures. To characterise the basin and provide physical parameters needed for the modelling, detailed geotechnical data were acquired. The basin infill consisted of up to $16 \mathrm{~m}$ of recent, fine-grained, water-saturated sediments, with shear-wave velocities increasing

\footnotetext{
1 Institute of Geological \& Nuclear Sciences, Wellington, New Zealand (Member)

2 Institute of Geophysics, Victoria University of Wellington, New Zealand (Member)
}

steadily with depth from $60 \mathrm{~m} / \mathrm{s}$ at the surface to $300+\mathrm{m} / \mathrm{s}$, underlain by sandstone basement, with a low shear-wave velocity of $850 \mathrm{~m} / \mathrm{s}$. As the results we present show, the lack of any boundary at which there is a very large abrupt change in shear-wave impedance, results in the seismic wave amplification being small compared with basins where large proportions of the incident seismic energy become trapped within the low-velocity layers. Another important feature of the results is that in no case did the incident $S$ wavefield contain simple coherent wavefronts. Instead, all the incident $S$ wavefields consisted of spatially-incoherent arrivals, probably due to scattering of the seismic waves by heterogeneities in the crust below the basin. Though this has meant that we were unable to verify the modelling using instances of precisely-known incident wavefields, we have reproduced statistical features of the observed amplifications, by modelling the response of the basin for ensembles of incident $S$ wavefields with similar time histories and random spatial distributions to the observed incident wavefields. This paper summarises representative results from the experiment, with the emphasis being on the stochastic nature of the incident $S$ wavefields for the weakmotion examples considered, and on the general character of the amplification. For full discussions of the geotechnical data and the experiment as a whole the reader is referred to respectively 
the report by Stephenson (1993) and the Ph.D. thesis of $Y u$ (1996), from which papers will be produced on technical aspects of the study. A description of the observational data is given also by $Y u$ and Haines (1994), following on from preliminary results presented by Haines et al. (1994).

The very low shear-wave velocities of the soft soil in Alfredton basin are typical of sites where very large amplifications have been observed. New Zealand examples include the basins at Porirua and Wainuiomata and the area of marine sediments overlying the gravels in the Hutt Valley (Taber and Smith, 1992), the vicinity of the new Museum of New Zealand site on the Wellington waterfront (Taber, 1993), areas of thick silt and peat in central Christchurch (Taber and Cowan, 1993), and river and marine sediments in Wairoa and downtown Gisborne (Barker, 1994). The classic example worldwide is the area of fine-grained deposits in what was a lake 500 years ago covering much of the present-day heart of Mexico City, and a close analogue to this in New Zealand is at Wainuiomata where a similar lake existed until recent times (Stephenson and Barker, 1992; Begg et al., 1993). Amplifications at such sites are generally restricted to parts of the incident wavefields in specific, narrow frequency bands determined by the local site conditions, and the amplification factors in these frequency bands range from, say, 10 to 40 or more, relative to the corresponding wavefields at basement rock sites. As we will show, the amplifications in Alfredton basin are much smaller than this, because of the lack of a boundary where there is a large contrast in shear-wave impedance. Our results in this regard are in accord with calculations for comparable 1dimensional models by Davis (1995).

On the other hand, the amplications at Alfredton are larger than have been typically observed in strong motion at soil sites throughout New Zealand: Zhao et al. (in prep.) have found, for example, that the peak ground accelerations at sites with soil more than $3 \mathrm{~m}$ thick are only 41 percent stronger on average than at other sites. In comparison, for sites from each class the individual peak ground accelerations are significantly more variable with the standard deviation being 1.5 times the average difference between the two classes. We will show that the variability in peak ground acceleration between the closely spaced basement sites at Alfredton is only slightly smaller than that found by Zhao et al. (in prep.) for much more widely spaced sites.

The other outstanding feature of the wavefields observed at Alfredton is the spatial incoherence seen at the basement sites which surrounded the basin. Observations of the coherence of incident wavefields have been made in few places worldwide, and most observations that have been made have been in specially chosen, hard rock areas where closely spaced arrays of seismometers have been deployed so that the testing of nuclear weapons can be monitored globally. In other words, these areas were selected because the incident wavefields were likely to be as simple, coherent and easy to interpret as was thought possible. Even so, it is not unusual for the wavefields to be incoherent at sites separated by more than half a wavelength, as we find to be the case at Alfredton for the small earthquakes which were recorded, and the major cause of the incoherence is known to be scattering of the incident waves by heterogeneities in the crust in the vicinity of the recording sites ( $W u$ and $A k i$, 1985). Alfredton is the first experiment in New Zealand where spatial coherency has been tested on a similar scale. Because the shear-wave speed of $850 \mathrm{~m} / \mathrm{s}$ in the basement at Alfredton is very much lower than the wave speeds in hard rock, the half- wavelength distances over which the wavefields are coherent at any given frequency, are much smaller. Other sites in New Zealand where the basement rock is likely to be similarly "soft" are distributed throughout the North Island, where much of the near-surface material is little more than compacted mud.

\section{THE ALFREDTON EXPERIMENT AND PHYSICAL PROPERTIES OF THE BASIN}

The site at Alfredton in the southern part of the North Island, New Zealand (Figure 1), is a small, roughly round sedimentfilled depression 400-500 $\mathrm{m}$ in diameter (Figure 2). The basin is bounded to the north by the Alfredton fault which has affected the flow of the Ihuraua River, to the west by a hill, to the east by highway 52 at the base of another hill, and to the south the basin widens out into a much larger, less boggy area of recent sediments.

The 19 identical EARSS portable seismographs from GNS (Institute of Geological \& Nuclear Sciences) and Institute of Geophysics, Victoria University of Wellington, were deployed initially at $100 \mathrm{~m}$ spacing (Figure 2). Towards the end of the experiment the instruments at outlying sites A13 and A18 were moved to new sites A19 and A20 to give denser coverage in the centre of the basin with $50 \mathrm{~m}$ spacing. Four of the seismographs were placed at firm, basement sites A00, A15, A11 and A12 surrounding the basin, and the remainder were placed at soft soil sites. Each of the instruments was independently triggered, and at almost all sites only the biggest ground motions during the 5 weeks of the experiment were recorded. In no event during this period was the ground motion large enough to be called "strong ground motion".

All earthquakes recorded by one third or more of the instruments were either at or near the base of the crust or were deeper Hikurangi Margin subduction events. 15 events (Figure 1) that were magnitude 2.5 to 5.1 and recorded by at least 12 instruments were selected for detailed analysis. Table 1 shows source and recording information for these 15 events.

The geotechnical investigation of the basin (Stephenson, 1993) consisted of cone penetrometry (CPT) and seismic cone penetrometry (SCPT) at over 20 sites, and two $\mathrm{P}$ wave, seismic refraction profiles spanning the basin north-south and east-west (Figure 3). In addition, samples were taken of soft soil for laboratory measurement of water content and plastic limits and of basement material for measurement of void ratio, density, water content and sonic $\mathrm{P}$ and $\mathrm{S}$ wave velocities. Figure 4 shows the $S$ wave vertical travel times versus depth for the 8 sites at which SCPT was performed in addition to CPT. All curves bend smoothly downwards, showing that the $S$ wave velocity increases with depth in a continuous fashion. As well, it can be seen that at shallow depths the $\mathrm{S}$ wave velocities are lowest in the centre and eastern half of the basin. This is where the CPT results and the seismic refraction profiles show that the Ihuraua River has in the past cut deepest into the basement sandstone. The critical physical parameters for the wavefield modelling of the "weak ground motion" were the basement $S$ wave velocity of $850 \mathrm{~m} / \mathrm{s}$, the shape of the basin, shown in Figure 5, and the continuous, 3-dimensional distribution of $\mathrm{S}$ wave velocities, interpolated between the SCPT sites. 


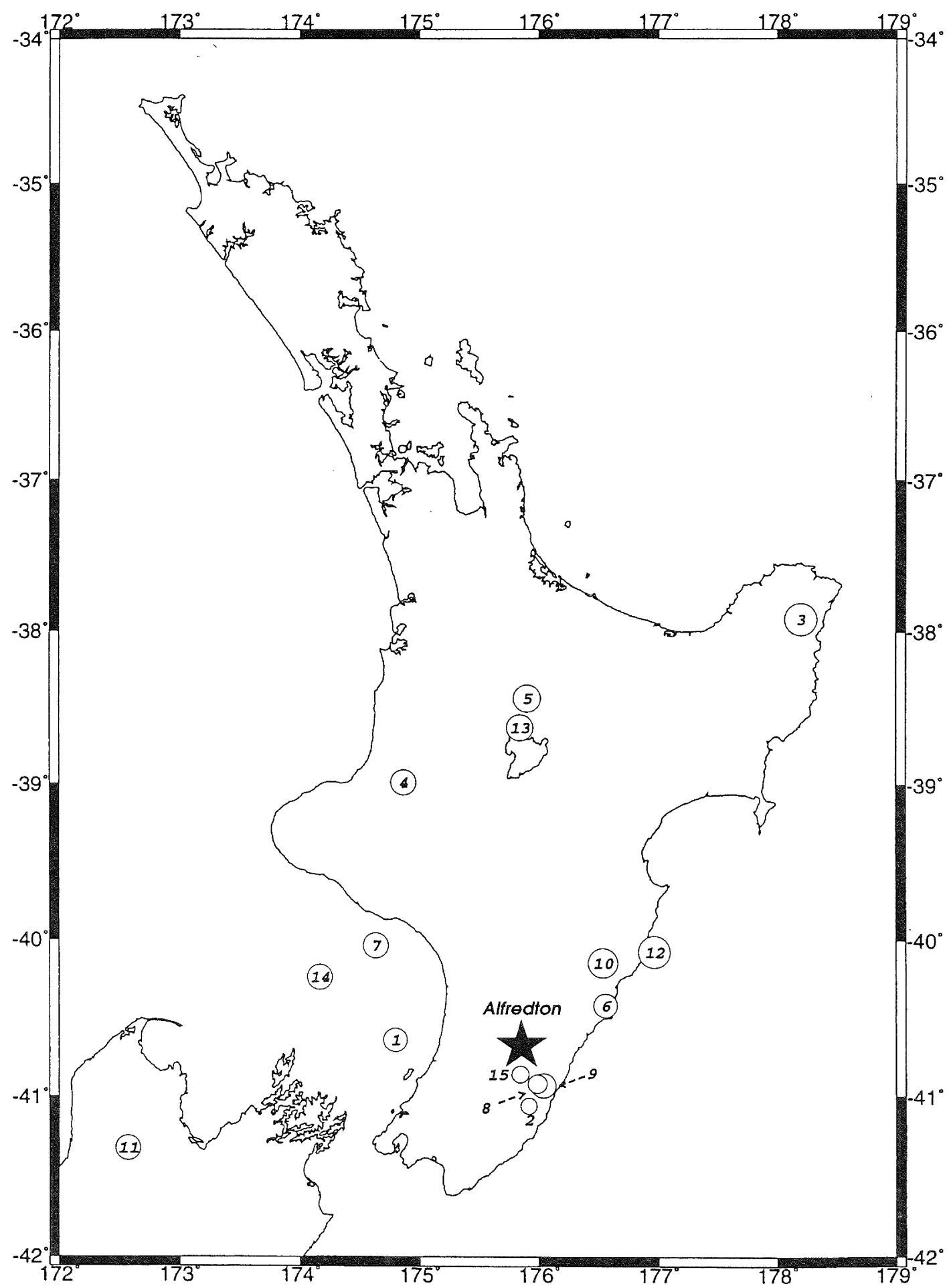

FIGURE 1. Location of Alfredton basin and the 15 earthquakes analysed in this study. 


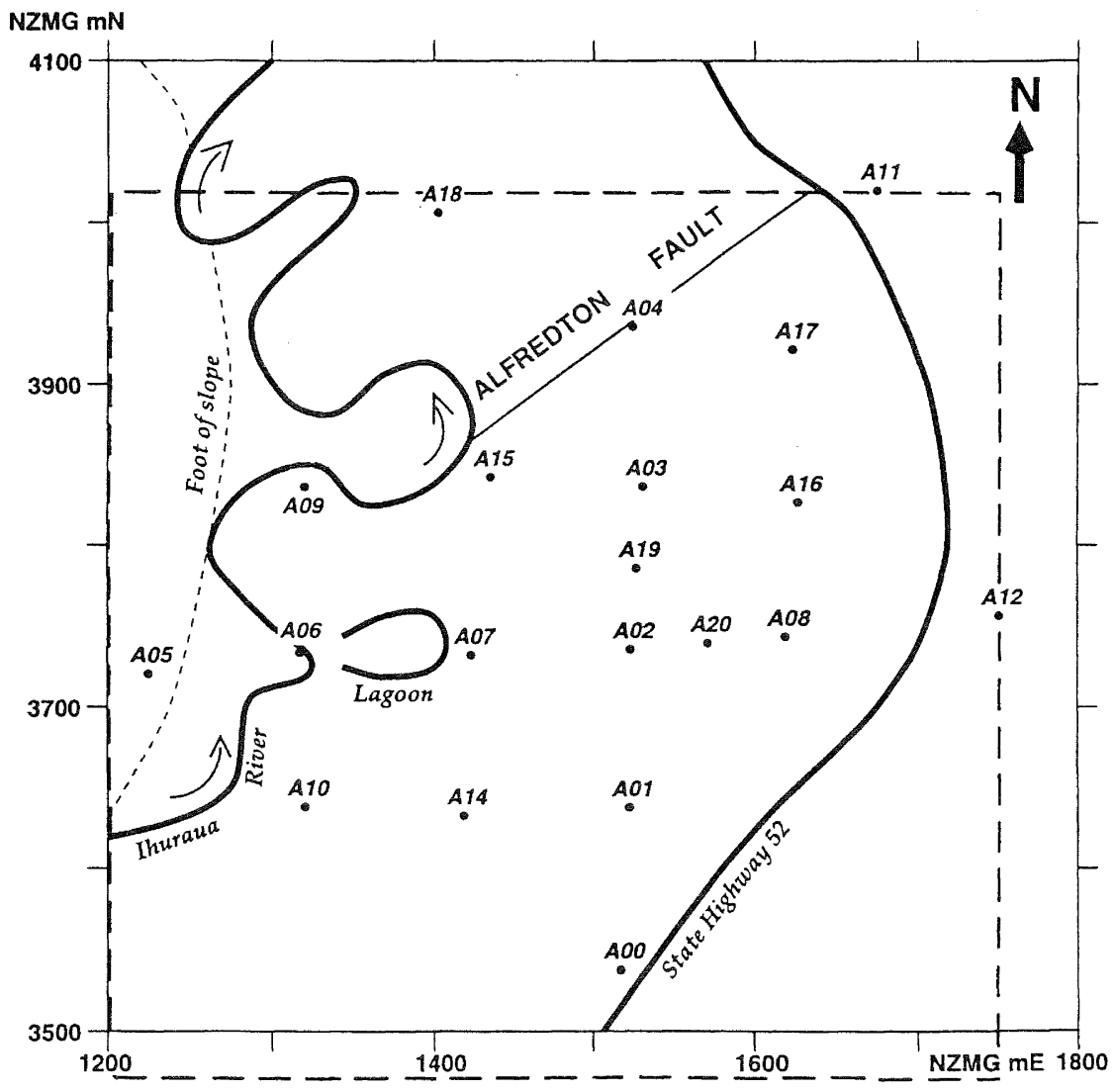

FIGURE 2. Location of seismometer sites. A00, A05, A11 and A12 are firm, basement sites. The square with the dashed outline is the area for which the geometry of the basin is shown in Figure 5.

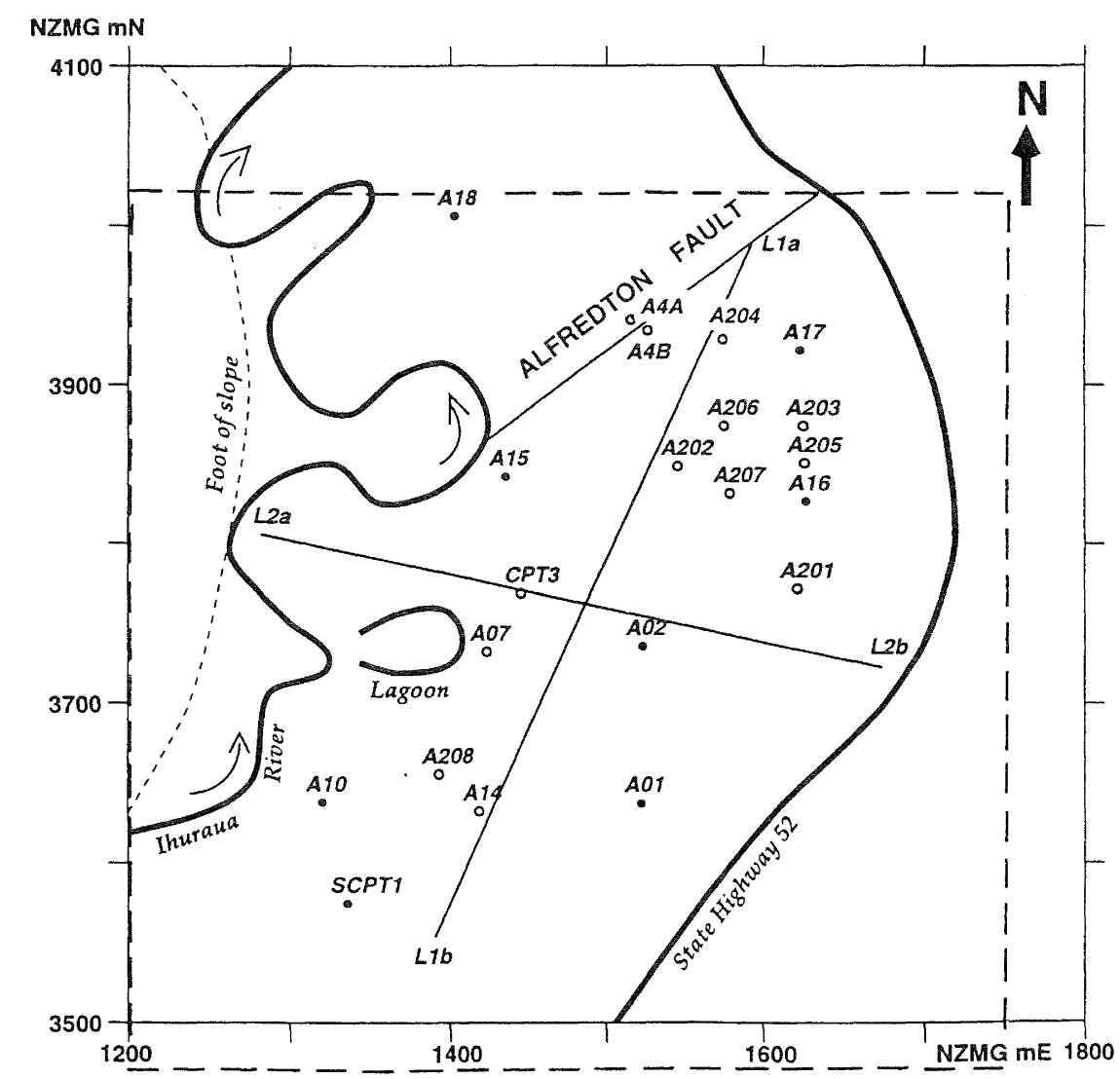

FIGURE 3. Location of penetrometer probes and seismic refraction profiles. Stars and circles denote respectively cone penetrometry (CPT) and seismic cone penetrometry (SCPT), which includes cone penetrometry as well. Solid lines mark the $P$ wave seismic refraction profiles L1 and L2, and the square with the dashed outline is the area for which the geometry of the basin is shown in Figure 5. 


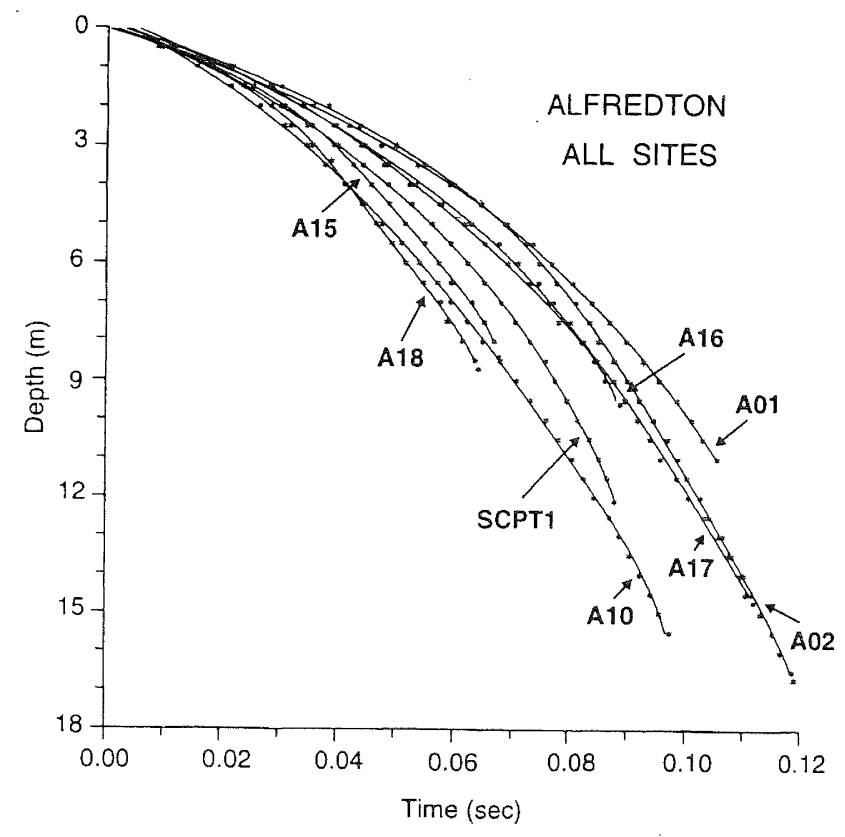

FIGURE 4. S wave travel times versus depth for the 8 sites at which seismic cone penetrometry data were acquired (from Stephenson, 1993). The shear-wave velocities, which are the gradients of the lines, are smoothly increasing with depth.

Topography of the basin

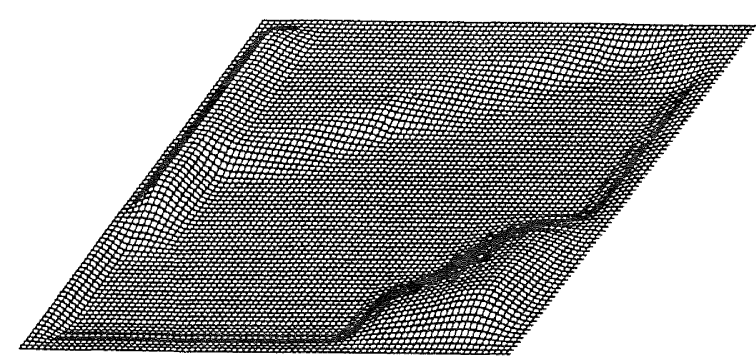

Bottom boundary of the basin

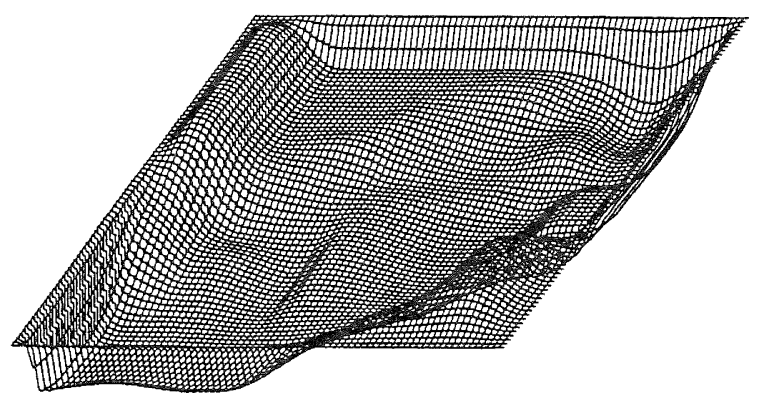

FIGURE 5. The geometry of the basin used in the wavefield modelling, showing the top and bottom surfaces, viewed from the south-southeast. The vertical exaggeration is 5:1. This is the area with the dashed outline in Figures 2 and 3.

\section{THE OBSERVATIONS}

In summarising the seismic wave observations we will focus first on aspects of the observed peak ground motions that are indicative of the incoherence of the incident wavefields and the patterns of amplification in the basin. We will show that two distinct patterns of amplification were observed. For the three events 8, 9 and 15 in Figure 1 that were closest to Alfredton the amplification in the basin was significantly larger than for the remaining 12 events we have studied in detail. In situations where there are large enough abrupt changes in shear-wave impedance to trap seismic energy in low-velocity, soft-soil layers, the duration of the ground shaking (number of cycles) is generally markedly increased in these layers, as well as there being increases in the amplitudes of the $\mathrm{S}$ waves (Borcherdt and Glassmoyer, 1992; Gutierrez and Singh, 1992). In contrast, at Alfredton we observed no noticable difference in duration between the motions in the basin and the motions at the four firm reference sites. We will illustrate that for the three events for which the amplification was largest the duration of shaking was very short, both at the reference sites and in the basin. Then for events in both this set and the other set we will show that the incident wavefields were incoherent except at low frequencies for which the diameter of the basin is less than half the wavelength of $S$ waves in the basement, much as has been observed elsewhere. We will conclude this section on the observations by presenting spectral ratios relative to the firm sites for a representative set of basin sites. These ratios illustrate that the spectral amplifications in the basin occur at frequencies higher than those at which the incident wavefields in the basement are coherent across the whole base of the basin

The $\mathrm{S}$ wave observational data presented in this paper, and by $Y u$ and Haines (1994) and $Y u$ (1996), are based on true ground motions recovered from the seismic recordings by allowing for the response of the instruments. However, all the results we will show have been normalised in some way, since the absolute levels of the motions are not important, given that the medium will have behaved linearly for the weak-motion strains involved. Unless we state otherwise, this will be with respect to the motion at the set of the four firm reference sites A00, A05, A11 and $\mathrm{A} 12$ that recorded each event. Because of the incoherence of the incident wavefields, we have had to normalise with respect to an average of the recordings at the reference sites, which we have generally chosen to be the appropriate RMS (root-mean-square) value.

Figure 6 shows the standard deviations of each of various measures of peak ground motion at the firm reference sites for each event, normalised with respect to the corresponding RMS value - D, V and A denote displacement, velocity and acceleration, and $\mathrm{Z}, \mathrm{E}, \mathrm{N}$ and $\mathrm{H}$ denote the vertical, east-west and north-south components and the maximum horizontal motion respectively. For comparison, for sites throughout New Zealand in separate rock and soil classes the corresponding standard deviation of peak ground acceleration found by Zhao et al. (in prep.) is equivalent to a value of 0.49 in Figure 6. It can be seen in Figure 6 that the differences between the peak values at the firm reference sites are greater for velocity than for displacement, and generally greatest for acceleration, for which the standard deviations approach the value found by Zhao et al. (in prep.) for much more widely spaced sites. This difference in variability between displacement, velocity and acceleration is in accord with the coherence of the incident wavefields decreasing with increasing frequency, given that peak displacement, velocity and acceleration are sensitive to the spectral amplitudes at progressively higher frequencies. No 

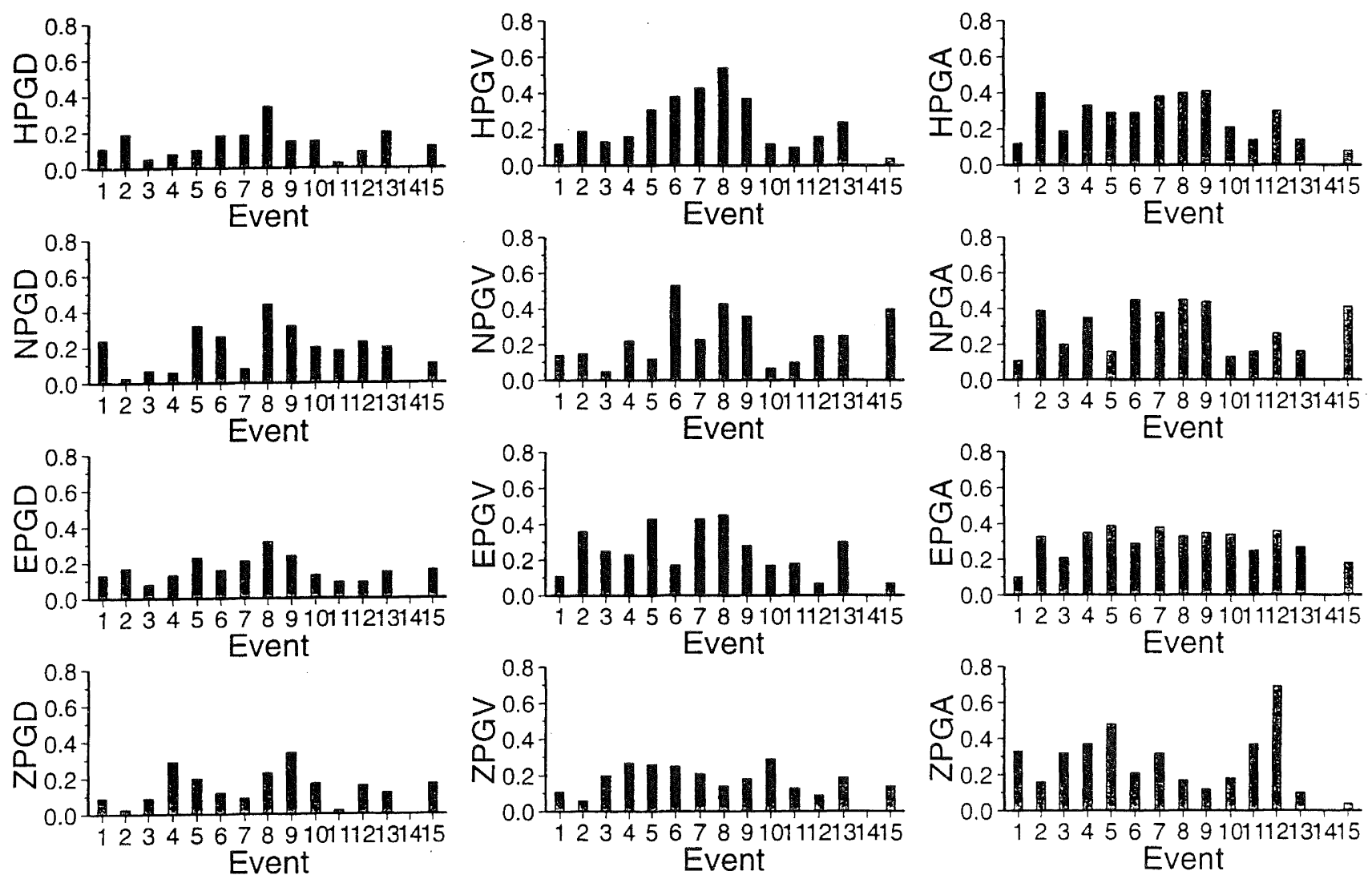

FIGURE 6. Standard deviations of the peak ground motion values at the firm sites recording each event. PGD = peak ground displacement, $P G V=$ peak ground velocity, $P G A=$ peak ground acceleration. $Z=$ vertical component, $E=$ east-west component, $N=$ north-south component, and $H=$ maximum horizontal motion. In each case, the normalisation is with respect to the RMS (rootmean-square) value, which is always greater than the standard deviation.

Table 1. Stations recording each event

\begin{tabular}{|c|c|c|c|c|c|c|c|c|c|c|c|c|c|c|c|}
\hline Event & 1 & 2 & 3 & 4 & 5 & 6 & 7 & 8 & 9 & 10 & 11 & 12 & 13 & 14 & 15 \\
\hline Lat S & 40.64 & 41.06 & 37.92 & 38.99 & 38.44 & 40.42 & 40.04 & 40.92 & 40.93 & 40.15 & 41.32 & 40.08 & 38.63 & 40.24 & 40.86 \\
\hline Long $E$ & 174.80 & 175.92 & 178.20 & 174.87 & 175.91 & 176.56 & 174.64 & 175.99 & 176.04 & 176.54 & 172.57 & 176.97 & 175.85 & 174.17 & 175.85 \\
\hline Depth & 46 & 31 & 59 & 195 & 157 & 23 & 107 & 27 & 27 & 46 & 197 & 40 & 143 & 104 & 30 \\
\hline Mag & 3.7 & 2.5 & 5.1 & 4.0 & 4.3 & 3.7 & 4.0 & 2.9 & 3.8 & 4.6 & 3.9 & 5.1 & 4.1 & 4.0 & 2.6 \\
\hline $\mathrm{A} 00$ & PS & * & PS & $S$ & PS & PS & PS & - & - & - & - & - & - & - & - \\
\hline $\mathrm{A} 01$ & * & * & PS & * & PS & PS & PS & PS & PS & PS & $\star$ & PS & PS & $S$ & PS \\
\hline $\mathrm{A} 02$ & PS & PS & PS & $*$ & $\mathrm{P}$ & PS & PS & PS & PS & PS & $P$ & PS & $\mathrm{P}$ & S & PS \\
\hline $\mathrm{A} 03$ & PS & PS & PS & $*$ & $P$ & PS & PS & * & PS & PS & PS & PS & PS & $S$ & PS \\
\hline $\mathrm{A} 04$ & - & $*$ & PS & $S$ & $\mathrm{P}$ & PS & PS & * & PS & PS & PS & PS & PS & S & PS \\
\hline AOS & PS & PS & PS & $S$ & $P$ & PS & PS & PS & PS & PS & PS & PS & PS & - & - \\
\hline A06 & PS & PS & PS & $S$ & $P$ & PS & PS & PS & PS & PS & PS & - & - & - & - \\
\hline A07 & PS & PS & PS & $S$ & PS & PS & PS & PS & PS & PS & $\mathrm{P}$ & PS & PS & $S$ & PS \\
\hline A08 & PS & PS & PS & * & $P$ & PS & PS & PS & PS & PS & $P$ & PS & $P$ & $S$ & PS \\
\hline A09 & PS & PS & PS & * & $P$ & PS & PS & PS & PS & PS & PS & PS & PS & PS & PS \\
\hline A 10 & PS & PS & $*$ & $S$ & * & PS & PS & PS & PS & PS & $*$ & PS & PS & $S$ & PS \\
\hline All & PS & PS & PS & $S$ & PS & PS & PS & PS & PS & PS & PS & PS & PS & $*$ & PS \\
\hline $\mathrm{A} 12$ & PS & PS & PS & $S$ & PS & PS & PS & PS & PS & PS & PS & PS & PS & PS & PS \\
\hline$A 13$ & - & PS & * & $S$ & PS & PS & PS & - & - & . & - & - & - & - & - \\
\hline A 14 & - & * & PS & $S$ & PS & PS & PS & PS & PS & PS & * & PS & $*$ & S & PS \\
\hline A 15 & PS & PS & PS & $S$ & PS & PS & PS & PS & PS & PS & PS & PS & PS & $S$ & PS \\
\hline A 16 & - & PS & PS & $S$ & PS & PS & $*$ & PS & PS & - & - & - & - & - & - \\
\hline A 17 & - & PS & PS & * & $\mathrm{P}$ & PS & PS & PS & PS & PS & PS & PS & PS & S & PS \\
\hline A 18 & - & PS & $*$ & * & $\mathrm{P}$ & PS & PS & - & - & - & - & - & - & - & - \\
\hline A 19 & - & - & - & - & - & - & . & PS & PS & PS & PS & PS & $P$ & $S$ & PS \\
\hline A.20 & - & - & - & - & - & - & - & PS & PS & PS & PS & PS & PS & * & PS \\
\hline
\end{tabular}

$$
\begin{aligned}
\text { Notation in the table: } & \text { PS P and S waves recorded } \\
\text { P P waves recorded } & * \text { No recording } \\
\text { S S waves recorded } & - \text { Not in operation }
\end{aligned}
$$



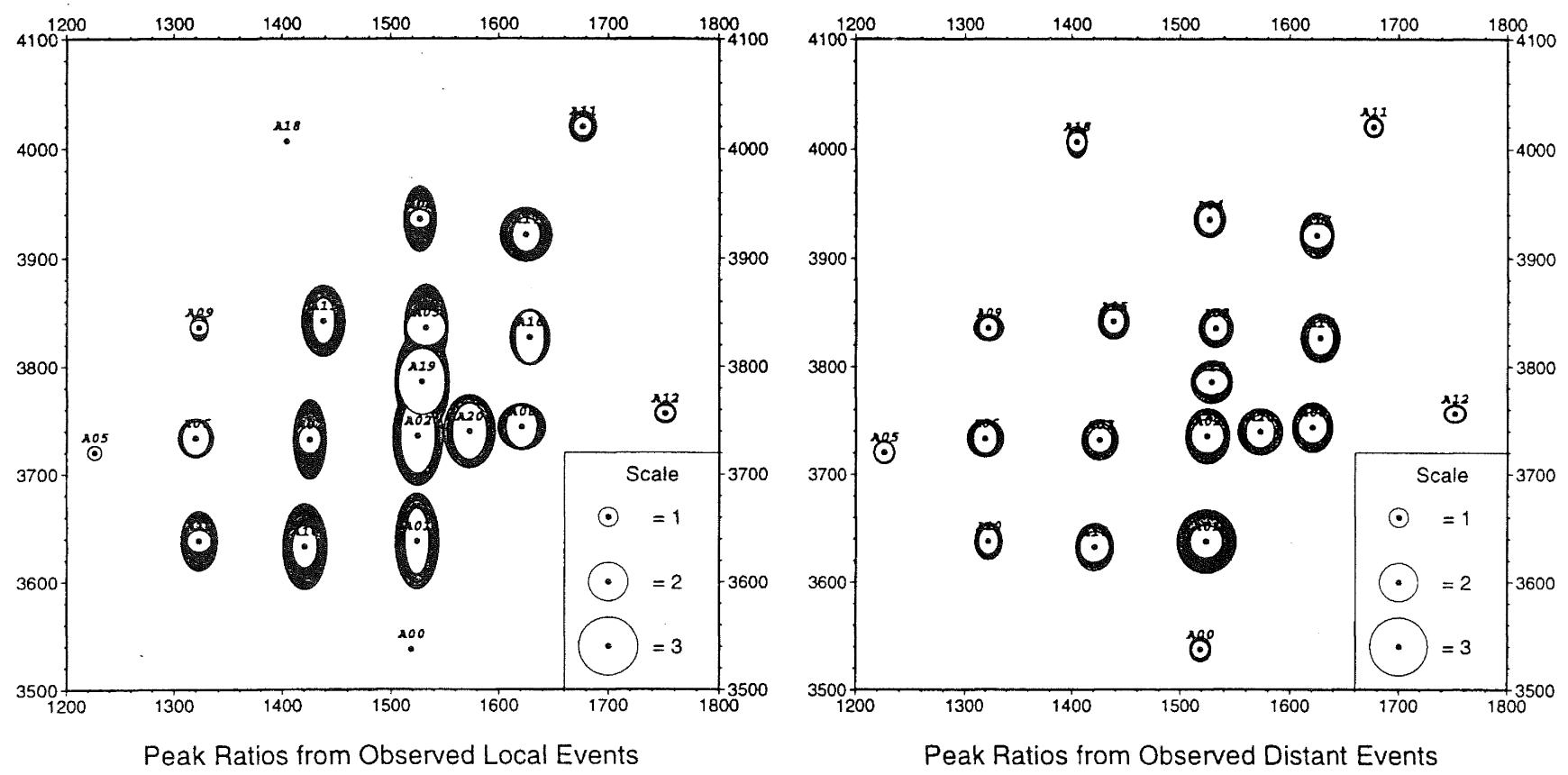

FIGURE 7. Statistics of the amplification of east-west and north-south horizontal peak ground displacement, for (a) the nearby events 8,9 and 15 and (b) the more distant events. Three ellipses are shown at each site, with axes in the east-west and north-south directions. The middle ellipse is such that the length of the axis in each of these two directions gives the mean value of the amplification factor, after the value for each event has been normalised with respect to the corresponding RMS value from the firm sites. The lengths of the axes of the outer and inner ellipses show the mean values plus and minus one standard deviation.

values are shown for event 14 because A12 was the only firm site that recorded that event (see Table 1). By normalising with respect to averages from all the reference sites for the other events, rather than with respect to the values at one site, say A12, we have obtained peak-motion and spectral amplifications in the basin that are significantly more consistent between events than they would have been otherwise.

Figure 7 summarizes the normalised east-west and north-south peak ground displacements at the individual sites, both inside the basin and at the surrounding firm sites. These are shown as ellipses with the axes in the east-west and north-south directions. Separate values are presented for the three special, local events and for the other, more distant events. The middle ellipse gives the mean normalised peak displacements in the two directions, averaged over the events recorded at each site, and the outer and inner ellipses are plus and minus one standard deviation.

As a guide in interpreting these results and the spectral ratios that are presented later, the following basic concepts are helpful. If absolutely none of the energy that enters the basin became trapped there (in the sense described below) and the incident $S$ wavefields were completely coherent, then the simplistic raytheory approximation for high-frequency wavefields ( $A k i$ and Richards, 1980) gives that the amplitudes in the basin would have been about 3.5 times the amplitudes at the firm sites. This approximation for situations where there are no abrupt changes in material properties is exactly the same as the high-frequency asymptote obtained by Davis (1995) for 1-dimensional models of this type that involve no damping. The difference in wavefield amplitude between soft and firm sites is dictated in such cases solely by the difference in material properties and is necessary for the energy flux to be the same, which depends on the product of the seismic impedance with the square of the amplitude of the wavefield - for $S$ waves the impedance is the product of the shear-wave velocity and the density, which is about $1.85 \mathrm{~g} / \mathrm{cm}^{3}$ in the basin and $2.2 \mathrm{~g} / \mathrm{cm}^{3}$ in the basement (Stephenson, 1993). If, instead, a large fraction of the energy were trapped in the basin for a long duration, the ratios of the peak amplitudes and, in particular, the spectral amplitudes at the dominant frequencies for the basin would be considerably greater than this value of 3.5 .

To clarify what we mean by "trapped" in the context we are considering, let us follow what happens to seismic energy after it has entered the basin. Initially the energy will propagate up to the surface. At the surface it will be reflected downwards. Then when it reaches the base of the basin, or any internal discontinuity where the impedance changes abruptly, a fraction of the energy will be reflected back upwards. How large this fraction is depends on the magnitude on the contrast in impedance at the discontinuity. If the contrast is very large, then almost all of the energy is reflected back upwards, which is why the word "trapped" is used. On the other hand, if the impedance below the discontinuity is, say, three times or twice the impedance above the discontinuity, much as is the case at Alfredton, the fraction of the energy that is reflected upwards is only one quarter or one ninth respectively. This process is then repeated until all the energy trapped in the basin has escaped.

The results in Figure 7 show that on the whole, rather than being greater than or equal to 3.5 , the normalised values of the peak amplitudes in the basin are more typically about 2 . Part of the explanation for this might be that weathering of the exposed basement material near the ground surface has resulted in the $S$ wave velocity at the firm sites being less than the value 
of $850 \mathrm{~m} / \mathrm{s}$ obtained from cores taken from under the basin. This would have the effect that the difference in amplitude between the firm sites and the basin would be smaller. All the same, it is clear that at most a modest fraction of the incident energy can be being trapped in the basin. The pattern of peak velocities is almost identical to that in Figure 7 for the peak displacements, whereas the normalised values of peak acceleration are more variable at each site, and are less systematically distributed between sites, though overall the normalised values of peak acceleration inside the basin are marginally smaller than the normalised values of peak displacement and peak velocity $(Y u$ and Haines, 1994; $Y u$, 1996).

The most striking feature in Figure 7 is the markedly larger relative amplitudes in the basin for the north-south component for the three nearby events 8,9 and 15 . For these events the mean normalised peak displacements in the north-south direction of up to a value of 4 in the centre of the basin, were twice the values in the east-west direction. As we illustrate below, the distinguishing feature of the these events is the very short duration of the $\mathrm{S}$ wavefield. In the discussion of the modelling results in the next section we show that for incoherent incident wavefields with similarly short durations, the peak displacements in the basin are generally larger than for incoherent incident wavefields of longer duration. However, the modelling results predict that averaged over a large number of distinct events there should be no noticable difference in this behaviour between the north-south and east-west components. Given that events 8,9 and 15 were very close together, it is possible that they were also very similar in all other regards, including their source mechanisms and rupture characteristics; that is, they may have been almost identical events, that just happened to give larger amplitudes in the basin in the north-south direction than the east-west direction, but there is no way of verifying this satisfactorily.

A point to note with regard to the different amplifications for events 8,9 and 15 compared with the other events is that angle of incidence is most unlikely to have been a major factor in this difference. Because of the very low S velocity of $850 \mathrm{~m} / \mathrm{s}$ in the basement below the basin, the angles of incidence for the basic S-wave arrivals for all of the events would have been no more than $10-15^{\circ}$ from vertical. Consequently, the fact that events 8,9 and 15 were close to being directly beneath the basin is not especially significant.

Figure 8 shows $\mathrm{S}$ wave horizontal ground displacements in one second segments for the largest nearby event 9 (magnitude 3.7) and event 12 (magnitude 5.1) which had the largest ground motions of the more distant events. The displacement paths are shown for the firm site A12 and two representative sites A02 and A15 in the basin, and are normalised to the largest displacements, which are at site A02. The short duration of the main motion in event 9, lasting no more than a second, is particularly clear. It is also obvious that, apart from occurring at the same times, the motions at the three sites are similar only in that they all appear to be completely random.

The coherence of the wavefields at the firm sites is quantified for the same two events in Figure 9. Coherence values close to 1.0 imply that the wavefields are effectively the same at all of the firm sites $(Y u, 1996)$. Values are given as a function of frequency for overlapping, 6-second long time windows, starting
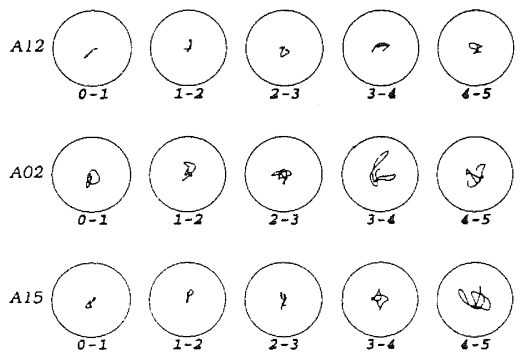

Tracks between $0-5$ seconds in event 12

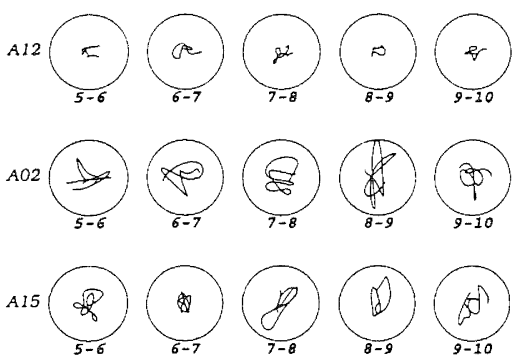

Tracks between 5-10 seconds in event 12
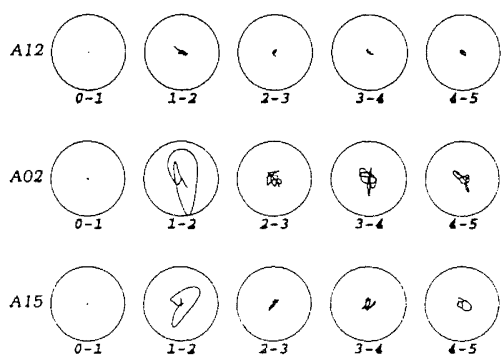

Tracks between $0-5$ seconds in event 9

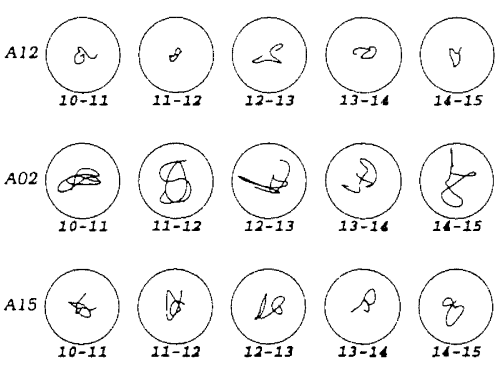

Tracks between 10-15 seconds in event 12

FIGURE 8. Horizontal ground displacements in events 9 and 12. Each window shows a track for one second. 

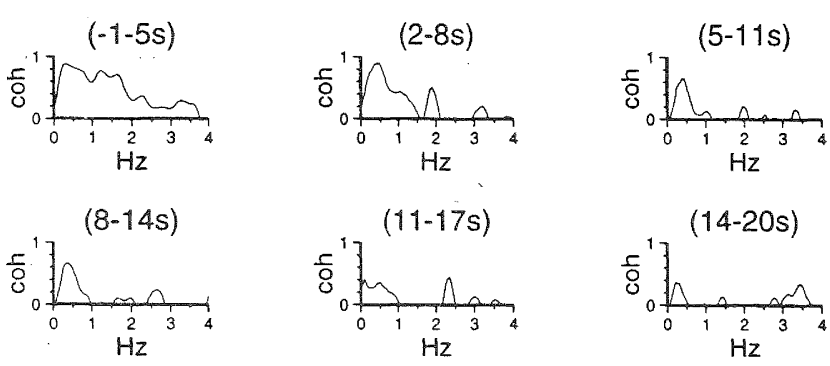

Event 9
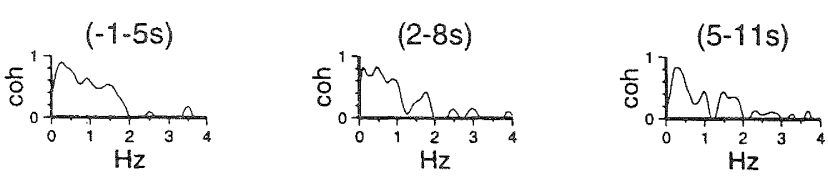

$(8-14 s)$
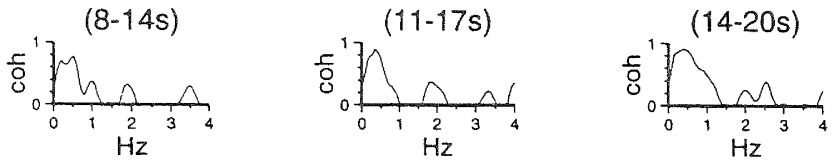

Event 12

FIGURE 9. Coherency versus frequency, for 6-seconds-long segments of the wavefields at the firm sites, for events 9 and 12 . The first segment starts 1 second before the arrival of the $S$ waves.

from the time of arrival of the $S$ waves. Each successive window begins 3 seconds after the start of the preceding window. A remarkable feature of the values in Figure 9 is that, as is true of all events, except the deep distant events $4,5,11$ and 13, the coherence patterns for events 9 and 12 are very similar, despite the obvious differences in Figure 8 between how the amplitudes vary with time for these two events. In the first time window for both events there is good coherence for frequencies up to $2 \mathrm{~Hz}$, but for the remainder of the wave trains there is significant coherence only for frequencies below $1 \mathrm{~Hz}$. A feature of the first part of the wave trains is that the angles of incidence at the base of the basin will have been close to vertical. Consequently, the effective wavelengths of the waves projected onto the Earth's surface will have been significantly longer than the actual wavelengths, but by uncertain amounts, and, as a result, it is necessary to use the later parts of the wavetrains, after the basic $S$-wave arrivals, to estimate the number of wavelengths along the Earth's surface over which the incident wavefields are coherent. Taking the average separation of the firm sites to be $400-450 \mathrm{~m}$, the $S$ wave velocity of 850 $\mathrm{m} / \mathrm{s}$ in the basement, and the upper frequency for coherence at the firm sites to be $1 \mathrm{~Hz}$, one gets that the incident wavefields are coherent over no more than half a wavelength. Given the similarity of the patterns in Figure 9 for events 9 and 12, how far away the earthquakes occurred is not significant in determining the correlation distance, which must, instead, be being dictated by scattering of the waves by heterogeneities in the crust below the basin, and possibly also by scattering from nearby surface terrain. The difference for the deep distant events $4,5,11$ and 13 , is that there was very little coherence at all in any part of the incident wavefields, even for frequencies below $1 \mathrm{~Hz}$.
The spectral ratios in Figure 10 for the north-south line of sites $\mathrm{A} 01, \mathrm{~A} 02, \mathrm{A0} 3$ and $\mathrm{A04}$, are the final observational data we will present here. It can be seen that the spectral ratios have similar characteristics to the peak ground displacements in Figure 7; that is, the spectral ratios for the east-west component for the three nearby events 8,9 and 15 are similar to the spectral ratios for both the east-west and north-south components for the more distant events, whereas the spectral ratios for the north-south component for the nearby events are generally larger. As well, it can be seen that the spectral ratios for the vertical component for three of the four sites shown are comparable in size to the ratios for the other components. For the two horizontal components the largest values of spectral ratio, of between 2 and 6 for the four, representative sites, are for frequencies in the range $3-4 \mathrm{~Hz}$.

\section{3-DIMENSIONAL MODELLING RESULTS}

The 3-dimensional seismic wave modelling was performed using the Riccati equation approach developed originally by Haines (1989) for perfectly-elastic waves and extended to general linear physical systems by Haines and de Hoop (1996). The corresponding computer codes for 2-dimensional weak-motion problems have been verified by comparing results for simple cases of 2-dimensional basins with solutions obtained using other techniques (Benites and Haines, 1991). This has not been possible for 3-dimensional problems, since no comparable computer codes exist for detailed analysis of the linear responses of small-scale basins. Instead, one of the aims of the experiment at Alfredton was to test the 3-dimensional modelling by finding examples among the observations for which the incident wavefields had simple, well-defined forms, that could be precisely interpolated between the firm sites surrounding the basin to obtain the exact incident wavefield everywhere beneath the basin. As it has turned out, for none of the observed events did the incident wavefield even remotely resemble this ideal case, and, given the uniform incoherence of all the observed incident fields, in hindsight it appears that there were unlikely ever to have been ideally-coherent weak-motion events at Alfredton. Thus, the experiment was unsuccessful in providing data to test the 3-dimensional modelling definitively. Even so, the modelling has been successful in reproducing many of the essentially-statistical features of the observations, as we will illustrate now.

Our Riccati equation approach works by simultaneously calculating the frequency-domain responses of the basin for a fundamental set of incident waves. Any incident wavefield can be constructed by summing multiples of the fundamental incident fields, and the corresponding response of the basin is obtained by summing the responses for the fundamental incident fields in exactly the same way. The fundamental incident wavefields we have used in modelling the response of Alfredton basin to incident $S$ waves are plane waves with both $\mathrm{SH}$ and $\mathrm{SV}$ polarizations. Figures 11 and 12 show the amplitude of the surface ground response for a suite of incident $\mathrm{SH}$ waves propagating from the east with frequencies $1 \mathrm{~Hz}, 3 \mathrm{~Hz}, 5 \mathrm{~Hz}$ and $7 \mathrm{~Hz}$. The responses for SV waves coming from the same direction are similar in character except that the east-west $(\mathrm{Ux})$ component of the response is dominant, whereas for the $\mathrm{SH}$ waves the north-south (Uy) component is the largest.

Features to be noted include the following. In these and the following three figures of the same type, all three components of motion are shown, including the vertical component (Uz) as well as the the horizontal components (Ux and Uy). For the 

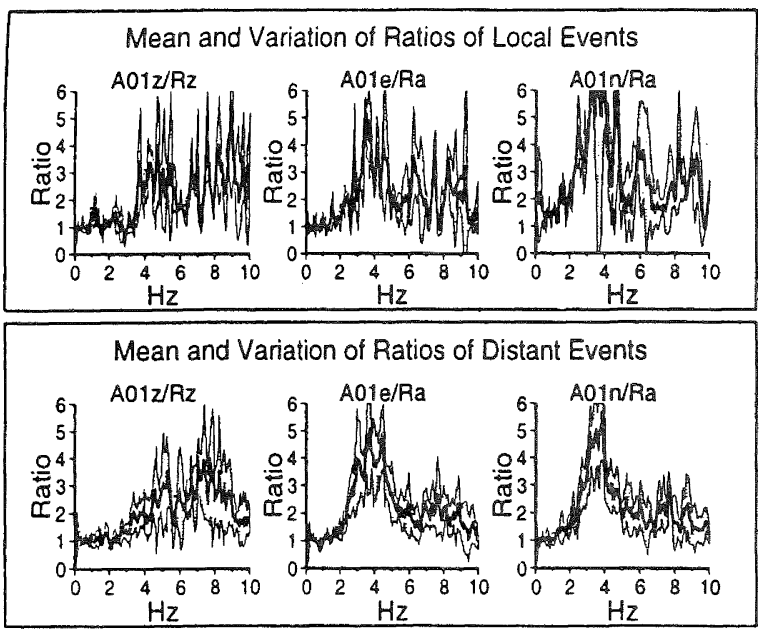

A01
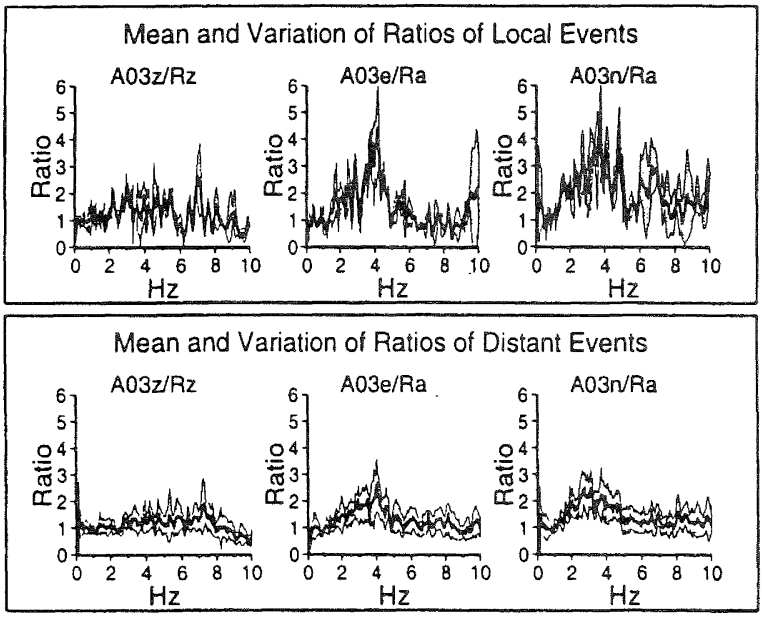

$\mathrm{A} 03$

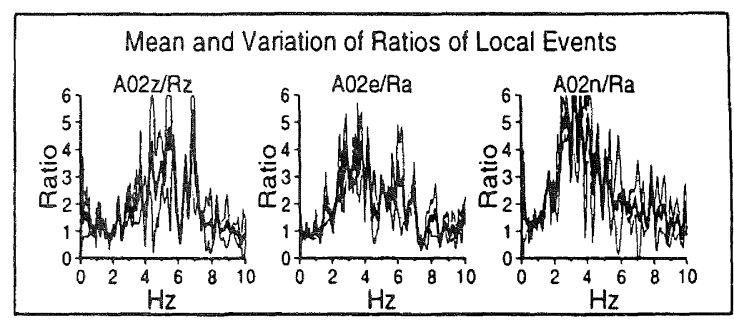

Mean and Variation of Ratios of Distant Events

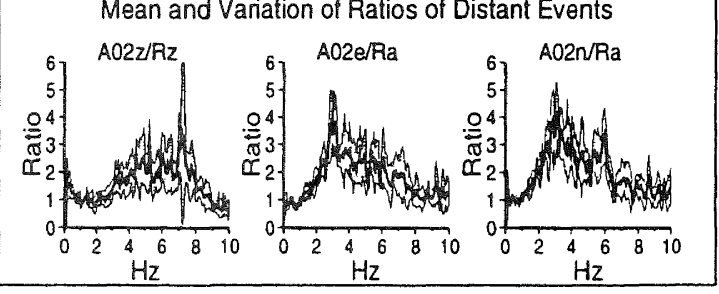

$\mathrm{A} 02$

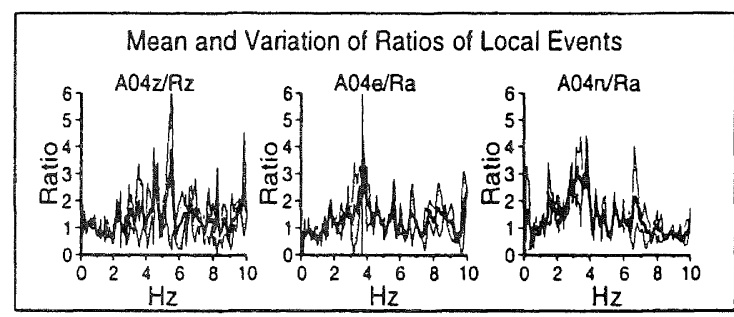

Mean and Variation of Ratios of Distant Events

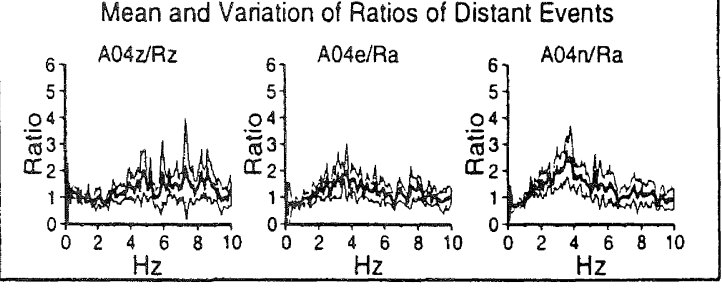

A04

FIGURE 10. Means and standard deviations of the Fourier spectral ratios at sites A01, A02, A03 and A04, with values shown separately for the nearby events 8,9 and 15 and for the more distant events. The east-west and north-south components have been normalised with respect to the joint $R M S$ value (Ra) of the east-west and north-south components at the firm, reference sites.

examples in Figures 11 and 12, though the incident SH waves involve only motion in the north-south direction, the other two components are activated in the resulting 3-dimensional response of the basin. In every case the amplitudes have been normalised by taking the amplitude of the incident wave to be 1.0 . Consequently, to convert these into values that can be compared with the observations, they have to be divided by 2.0 , since this is what the amplitude of the motion at the Earth's surface would have been for the SH case in the absence of the basin and surface topography. As a guide to how large the amplitudes are in these figures, the scale on the left-hand side of each plot has been set to the maximum amplitude plus 7.0, with the exception that a fixed value of 9.0 is used for maximum amplitudes below 2.0. For example, the largest amplitude of the north-south component (Uy) for the $3 \mathrm{~Hz}$ case in Figure 11 is approximately 12.0, which plus 7.0 is equal to 19.0 on the scale. After dividing by 2.0 to compare it with the observations, it becomes approximately 6.0. This is very similar to the largest values of the observed spectral ratios at $3 \mathrm{~Hz}$ in Figure 10.
Overall, the amplitudes of the north-south component in Figures 11 and 12 are comparable to the spectral ratios in Figure 10, and especially to those for the nearby events. At $1 \mathrm{~Hz}$ the presence of the basin has only a small influence on the amplitudes at the surface in Figure 11 which are close to 2.0 everywhere for the north-south component. This coincides with the observed spectral ratios all being close to 1.0 at $1 \mathrm{~Hz}$ in Figure 10. At $3 \mathrm{~Hz}$ there is a broad, continuous area where there is amplification, with the largest amplitudes being in the centre of the basin where sites A01 and A02 in Figure 10 are located (Figure 2). The largest amplitudes are slightly less at 5 $\mathrm{Hz}$ and $7 \mathrm{~Hz}$ in Figure 12, and the areas where amplification occurs are less continuous.

The remaining three figures of this type are all for wavefields at $3 \mathrm{~Hz}$. Figure 13 shows the amplitudes of the responses for the $\mathrm{SH}$ waves incident from the south and the north the same angle of incidence as for the $3 \mathrm{~Hz} \mathrm{SH}$ waves incident from the east in Figure 11. The major component of motion in these two 


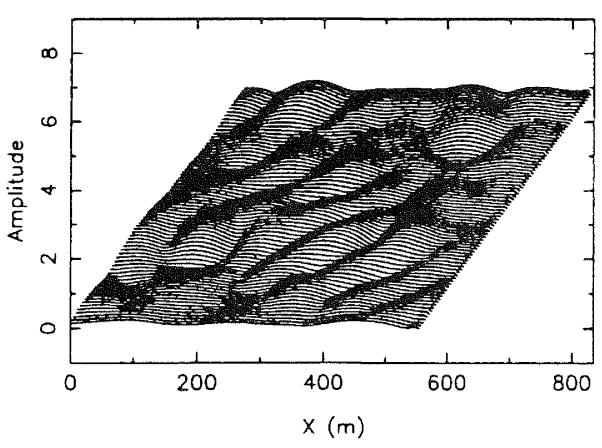

Uz

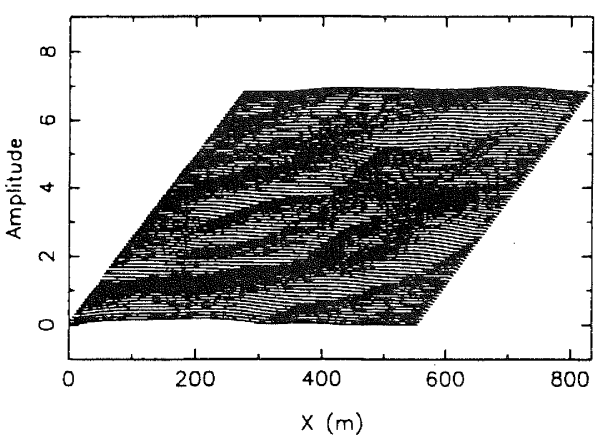

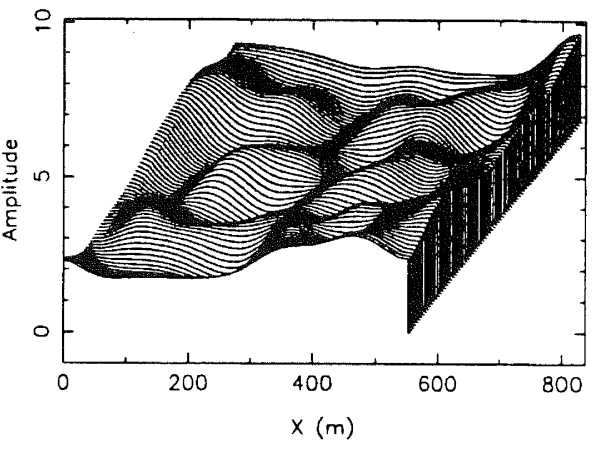

Level 0 of Alfredton Bosin

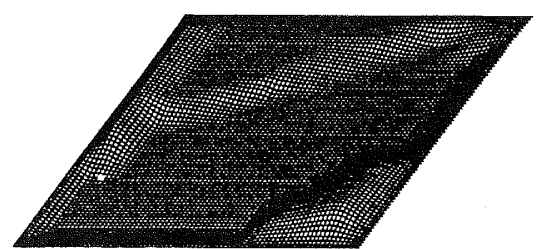

Ux

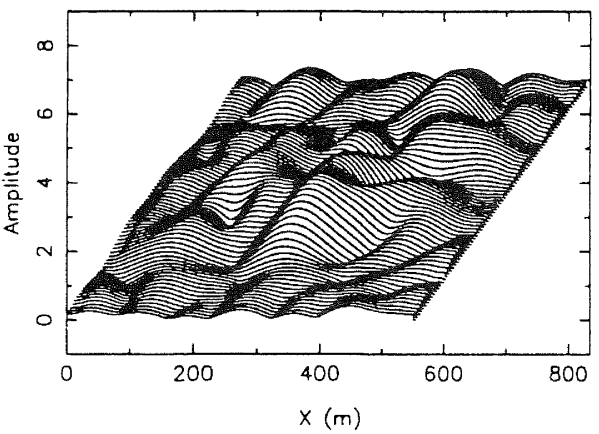

$\mathrm{Uz}$

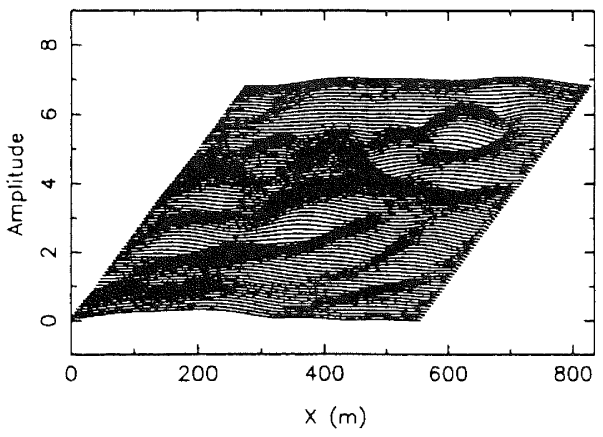

Uy

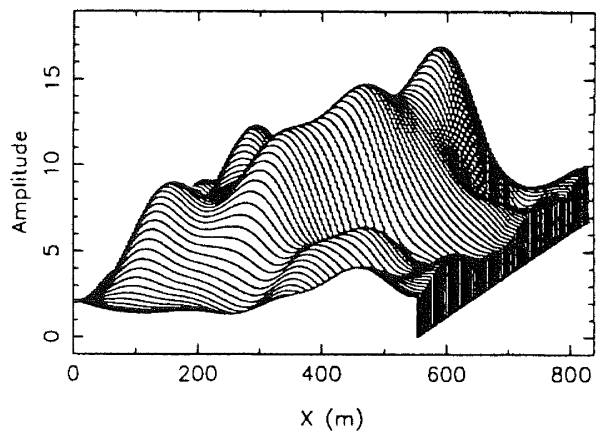

Level 0 of Alfredton Bosin

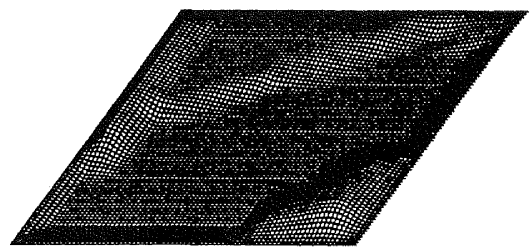

FIGURE 11. Amplitude distributions at the ground surface of the east-west (Ux), north-south (Uy) and vertical (Uz) components of the fundamental wavefields responding to incident Love waves of $1 \mathrm{~Hz}$ (top) and plane incident $\mathrm{SH}$ waves of $3 \mathrm{~Hz}$ (bottom) with an incident angle of $25^{\circ}$. Both incident wavefields propagate from the east, and the amplitudes, here and in Figures 12 to 15 , are normalised with respect to the amplitudes of the incident waves, which for $\mathrm{SH}$ waves in the idealisation of a flat uniform halfspace, are half the size of the total wavefields at the surface. The wavenumbers $(k x, k y)$ in the heading for each set of plots give how many cycles of the incident waves there are across the base of the area where the wavefield has been modelled. Negative and positive values of $k x$ indicate propagation from the east and west respectively, while negative values of ky indicate propagation from the south and positive values indicate propagation from the north. 
Ux

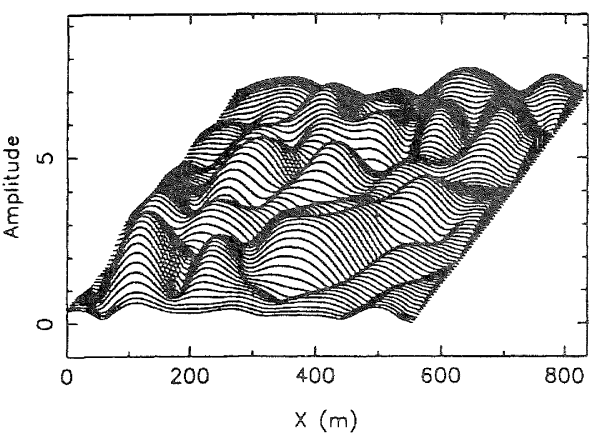

Uz

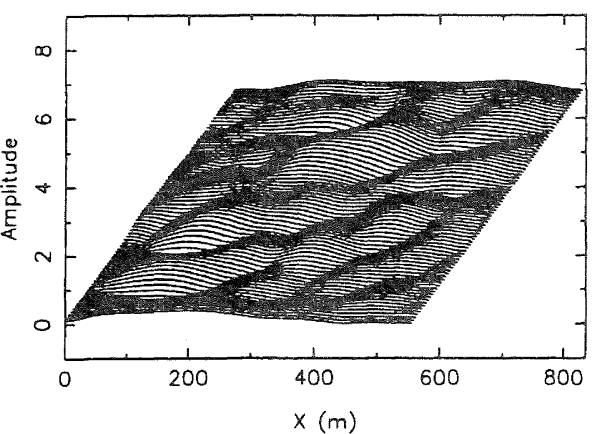

Uy

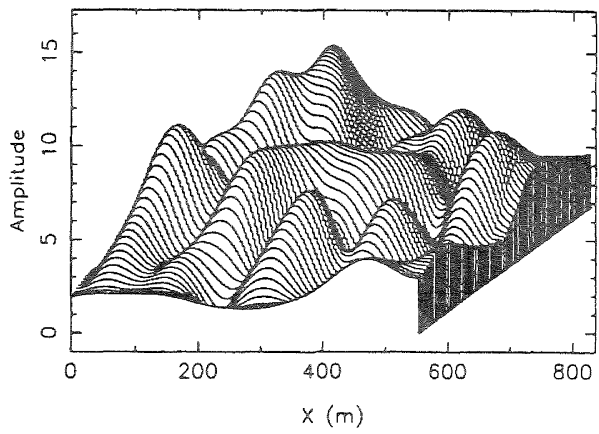

Level 0 of Alfredton Basin

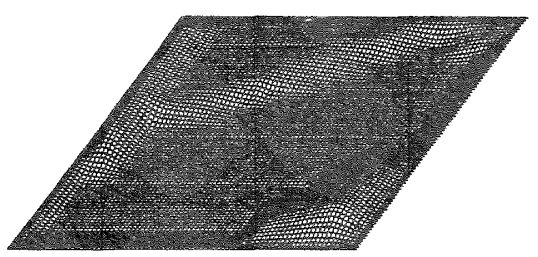

$u x$ SH inc. with $(k x, k y)=(-1,0)$, freq $=7.00$

Uy

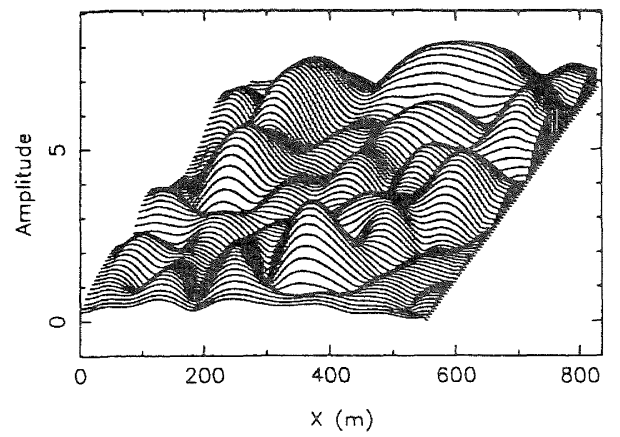

$\mathrm{Uz}$

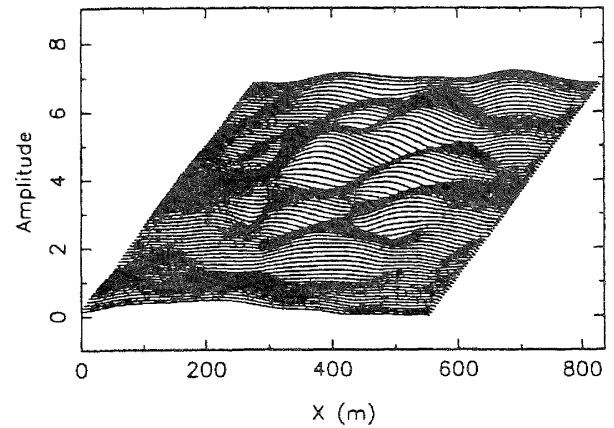

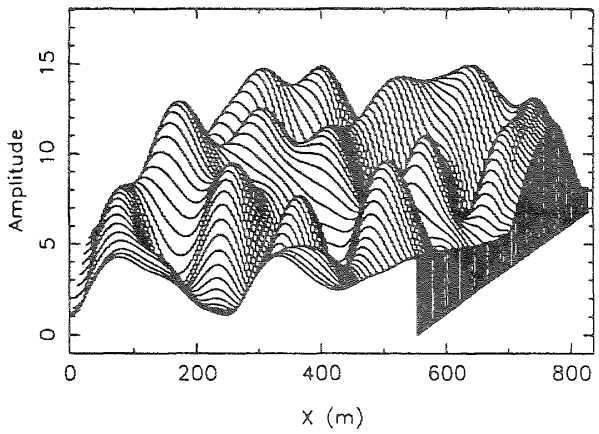

Level 0 of Alfredton Basin

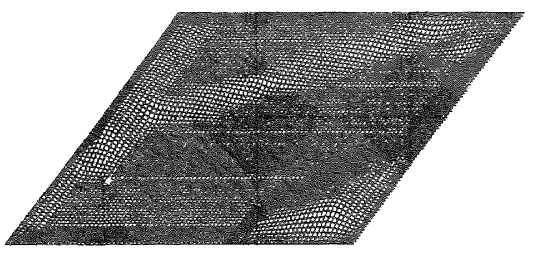

FIGURE 12. Amplitude distributions at the ground surface of the fundamental wavefields responding to plane incident $\mathrm{SH}$ waves of $5 \mathrm{~Hz}$ (top) and $7 \mathrm{~Hz}$ (bottom) propagating from the east with incident angles of $15^{\circ}$ and $10^{\circ}$ respectively. 
Ux

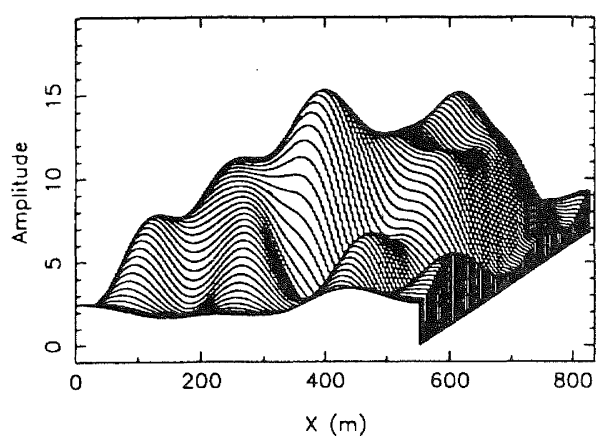

$\mathrm{Uz}$

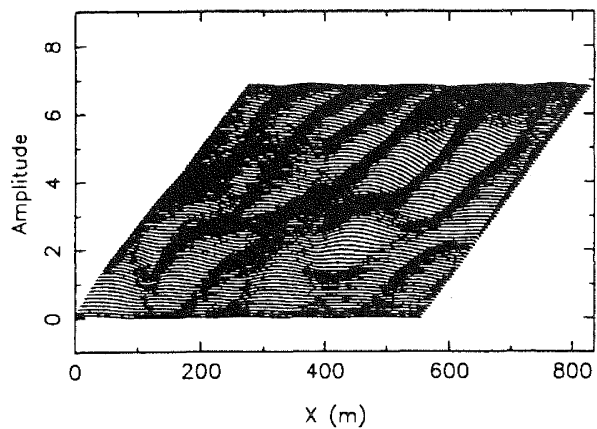

Uy

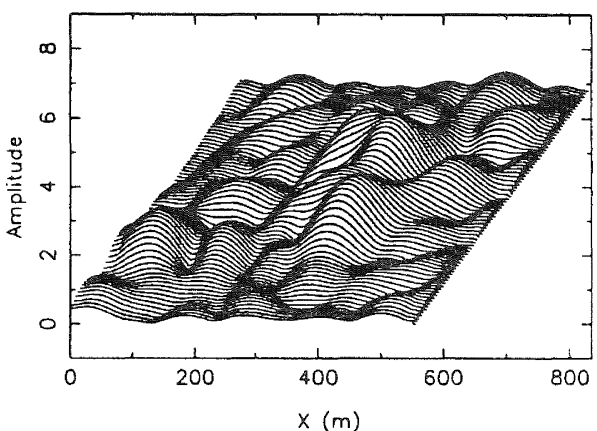

Level 0 of Alfredton Basin

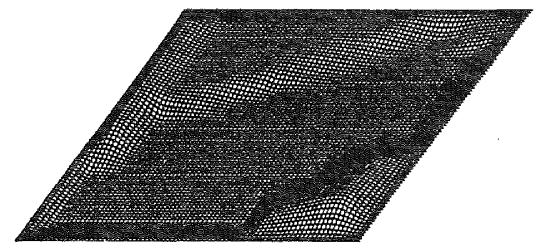

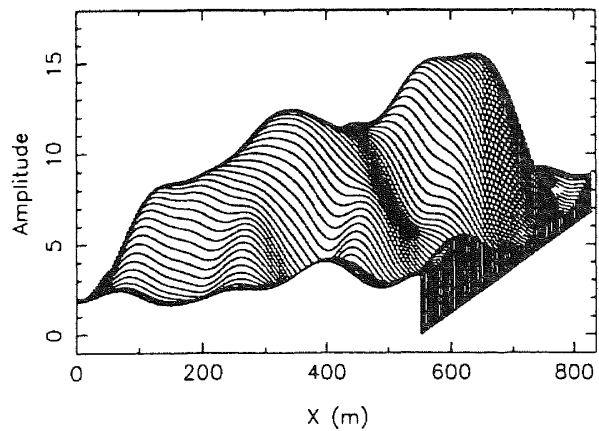

Uz

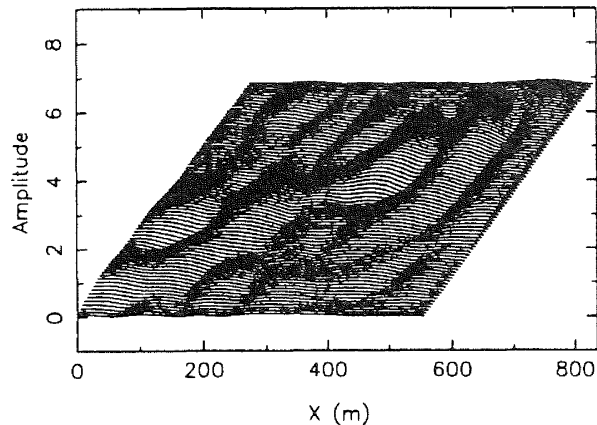

Uy

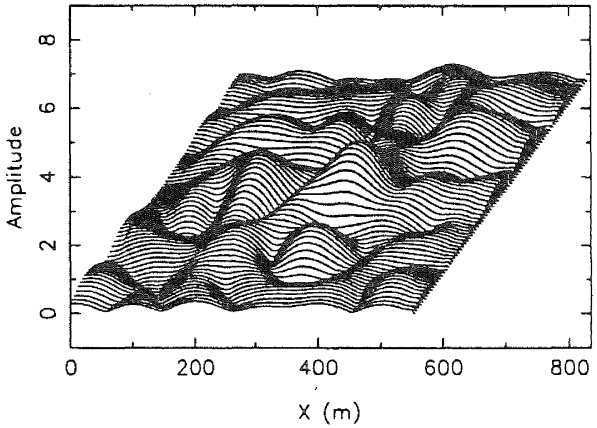

Level 0 of Alfredton Bosin

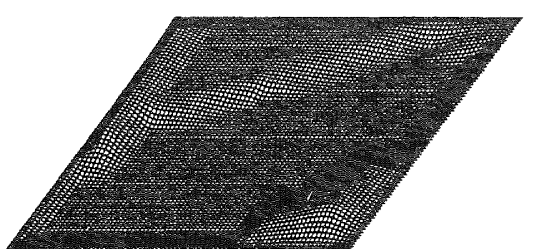

FIGURE 13. Amplitude distributions at the ground surface of the fundamental wavefields responding to plane incident $\mathrm{SH}$ waves of $3 \mathrm{~Hz}$ propagating from the south (top) and north (bottom) with the same incident angle of $25^{\circ}$ 
cases is in the east-west direction. Otherwise, the patterns are broadly similar to that in Figure 11, but at any given point the amplitude of the major component has different values in all three cases. A feature to note in all these examples is that the amplitudes are low at the southern end of the basin because the basin has been artificially terminated there, as is shown in Figure 5. In Figures 11 and 13 the angle of incidence for the $3 \mathrm{~Hz}$ waves is $25^{\circ}$ to the vertical. The incident waves for the cases in Figure 14 are horizontally propagating from the same geographical directions as in Figure 13. At most points the amplitudes in Figure 14 are much smaller than for the cases in Figure 11 and 13

In computing the response inside the basin, the wavefield has been expressed as the superposition of depth-dependent contributions from a preset number of horizontal wavenumbers. For the results presented thus far there were 69 horizontal wavenumbers. The number of horizontal wavenumbers determines how long the computations take. As well, there is spatial smoothing inherent in using expansions in terms of wavenumbers. 69 was chosen as the minimum number that would give an adequate representation of the variability of the wavefield between different sites. With this number of horizontal wavenumbers, the wavefields and the geometrical and physical parameters of the layers in the basin are averaged horizontally within each layer over an area approximately of radius $70 \mathrm{~m}$ about each point - in the calculations using the Riccati equation approach, there is no smoothing in the vertical direction. This horizontal resolution is sufficient to give separate results for each recording site, except where the recording sites were only $50 \mathrm{~m}$ apart, and is comparable to the spacing in Figure 3 at which probing was performed to determine the properties of the basin. Figure 15 shows the amplitudes of the surface ground response for the same $3 \mathrm{~Hz}$ incident SH wavefield as in Figure 11, except that 109 (top) and 145 (bottom) horizontal wavenumbers have been used. In these cases the approximate radii for the horizontal averaging are 60 $\mathrm{m}$ and $50 \mathrm{~m}$ respectively, and the computations took roughly 4 and 9 times as long. The patterns in Figure 15 are very similar to that in Figure 11, though the extra smoothing in Figure 11 is clearly evident. The time-domain wavefields described below were computed using the set of 69 horizontal wavenumbers, for the obvious reason of computation time, given the huge number of calculations involved.

To provide modelling results that can be compared directly with, for example, the peak motion statistics in Figure 7 for the observations, suites of synthetic "earthquakes", for which ground motions were computed in the basin and at the surrounding firm sites, were generated in the following manner. First, solutions were obtained for the wavefields in and around the basin generated by the 69 different SH waves and 69 different $S V$ waves in the fundamental set of incident wavefields for each of 120 uniformly spaced frequencies up to $12 \mathrm{~Hz}$ (giving 10 seconds of synthetic record for the comparisons) the number of incident wavefields in each fundamental set, which includes $\mathrm{P}$ waves as well, matches the number of independent 3-dimensional responses calculated for the basin, which is determined by the number of horizontal wavenumbers used in computing the wavefields. In other words, 16,560 independent, 3-dimensional, single-frequency, 3-component wavefield solutions, like the 8 in Figures 11 to 14 , were computed for incident $S$ waves.

Next, to mimic the incident waves for an actual earthquake, randomly chosen multiplying factors, or weights, were selected for the incident wavefields in the fundamental set for each frequency. The weights were chosen so that the composite incident wavefield obtained by summing all the contributions would have similar spatial coherency properties (Figure 9), angles of incidence and time histories, to a given set of observed ground motions at the firm reference sites, whose time envelopes and frequency spectra were used as templates in generating the random numbers used as the weights. Then, the responses for all the fundamental incident wavefields were summed, multiplied by these weights, to give the total timedomain response for the synthetic earthquake. This was repeated several times, keeping the same observed earthquakes as models, with the result that different synthetic events with similar time histories were generated with randomly different combinations of $\mathrm{SH}$ and $\mathrm{SV}$ waves and combinations of azimuths of arrival. For any given synthetic event the displacement paths of the ground motions for the incident waves were random functions of time, just like the observed displacement paths in Figure 8

Figure 16 summarizes the normalised east-west and north-south peak ground displacements at individual sites for two sets of synthetic events, in the same way that Figure 7 summarizes the observations. The normalisation for the synthetic results was with respect to the RMS of the peak ground displacements at the four basement sites in Figure 16 closest to the reference sites for the observations A00, A05, A11 and A12. One set of symthetic events all have time envelopes similar to that in Figure 8 for the largest nearby event 9 , with the frequency contents also being much the same as for event 9 . The other set are similar to event 12 , which is also shown in Figure 8 and had the largest ground motions of the more distant events. For the synthetic "nearby" events with short durations of the incident $S$ wavefields, the normalised peak horizontal displacements in the basin are generally larger than for the synthetic "more distant" events with longer durations, reflecting the fact that for shortduration events there is less opportunity (i.e. a smaller window in time and space) for the incoherent wave packets inside the basin to overlap and destructively interfere. The patterns and values in Figure 16 are clearly similar to those in Figure 7, except that in Figure 7 the amplitudes for the nearby and more distant earthquakes are noticably different only for the northsouth component. As already remarked, this might have been a chance occurrence, with all the nearby events being almost identical events that happened to give larger amplitudes in the basin in the north-south direction than in the east-west direction. The individual synthetic "nearby" events, on the other hand, have quite different incident wavefields, effectively covering a wide range of possible source mechanisms, with the result that on average the amplitudes are similar in the north-south and east-west directions.

\section{CONCLUSIONS}

The 3-dimensional modelling confirms that in the Alfredton basin the peak ground motions are more amplified for events with short duration incident wavefields than for longer duration events. This result appears to be an interesting curiosity, rather than having a direct bearing on seismic hazard, since 3.7 was the magnitude of the largest nearby earthquake for which the ground motion was of short duration. For large nearby events the duration of the ground motion is likely to be much longer. and, consequently, the amplification of the peak ground motions may be more like that observed and modelled for more distant events. This is not, however, a firmly based conclusion, as it is uncertain whether for earthquakes large enough to be relevant in assessing seismic hazard, the incident wavefields will be as 
Ux

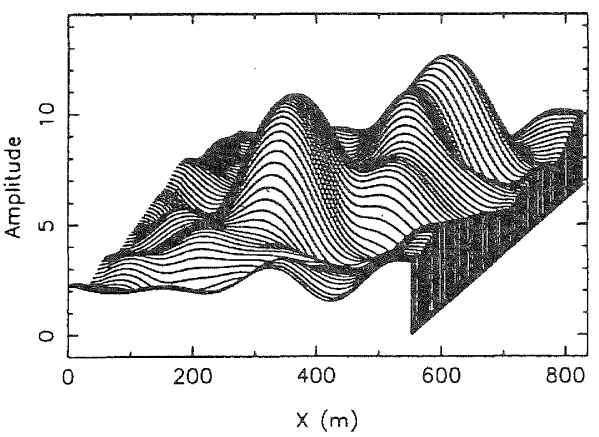

Uz

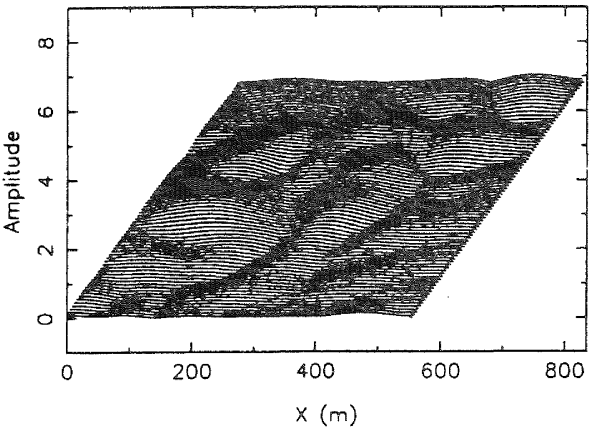

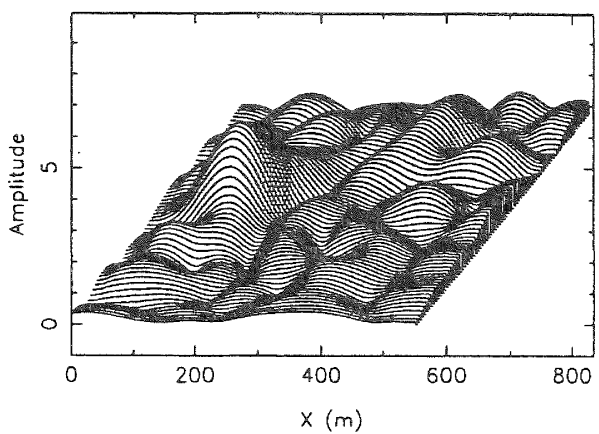

Level 0 of Alfredton Basin

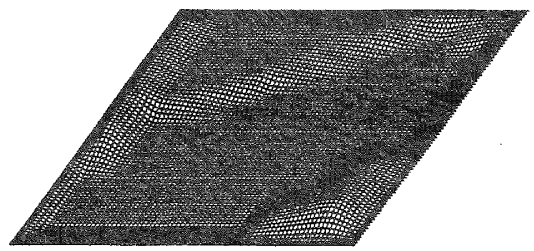

Ux

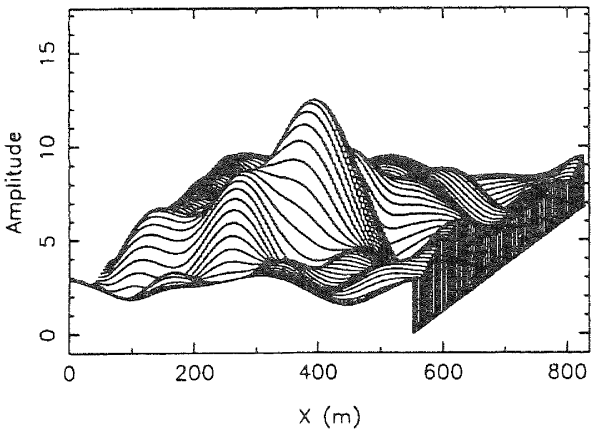

Uz

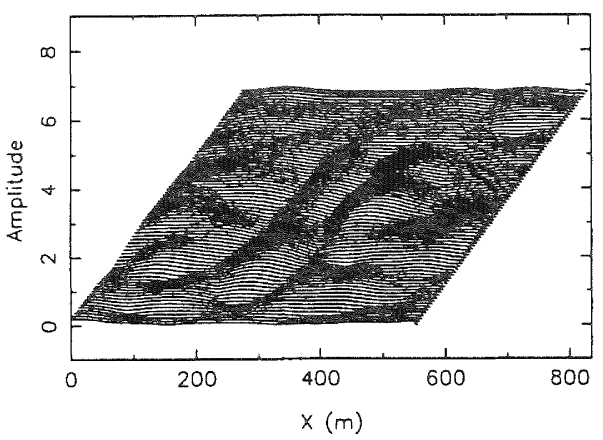

Uy

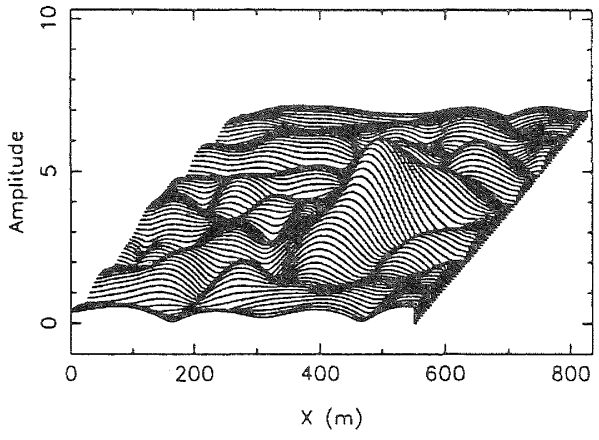

Level $O$ of Alfredton Bosin

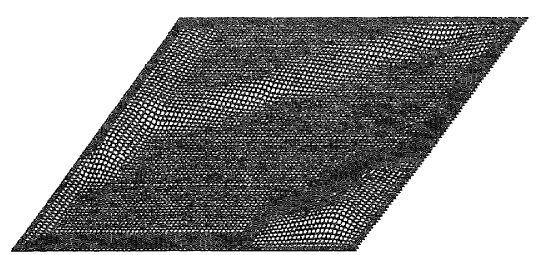

FIGURE 14. Amplitude distributions at the ground surface of the fundamental wavefields responding to incident Love waves of $3 \mathrm{~Hz}$ propagating from the south (top) and north (bottom). 


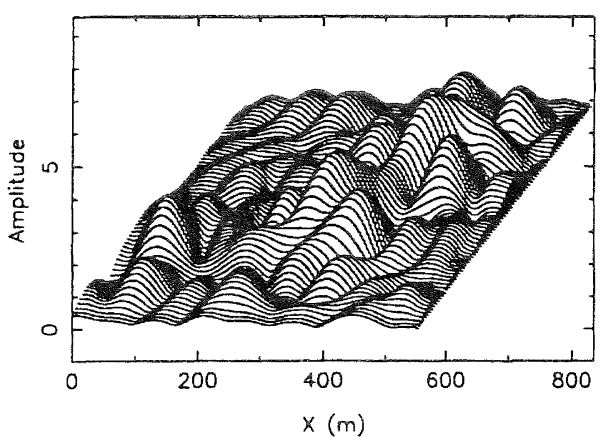

$\mathrm{U}_{2}$

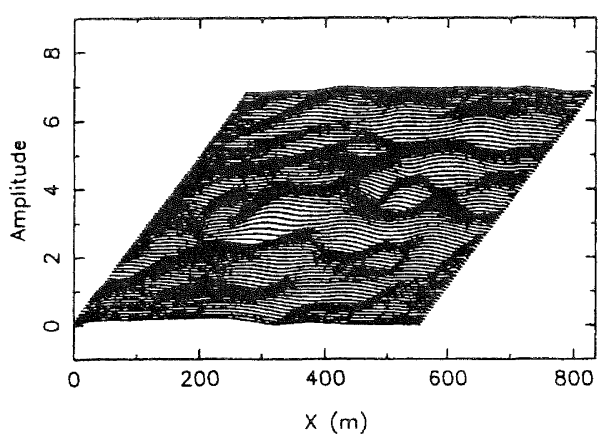

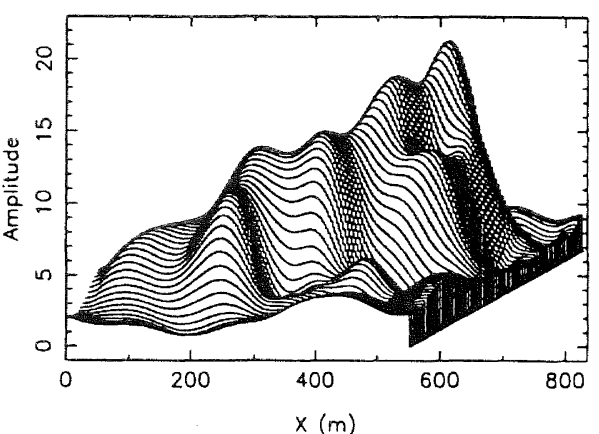

Level $O$ of Alfredton Basin

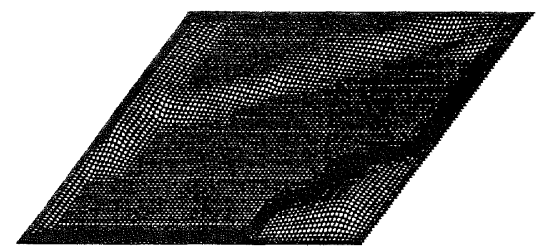

$U_{x}$

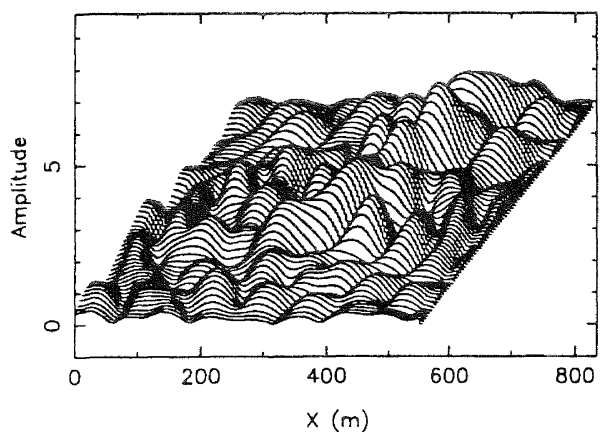

Uz

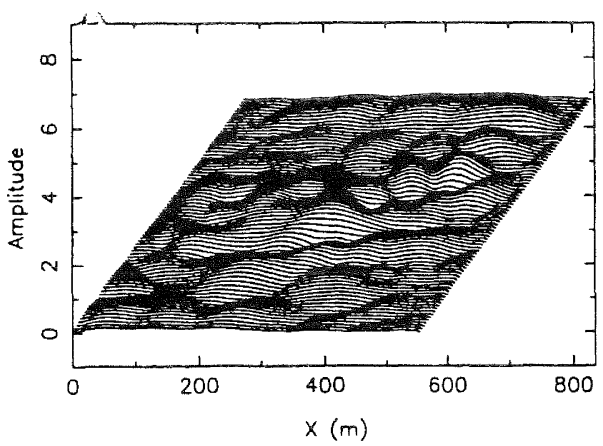

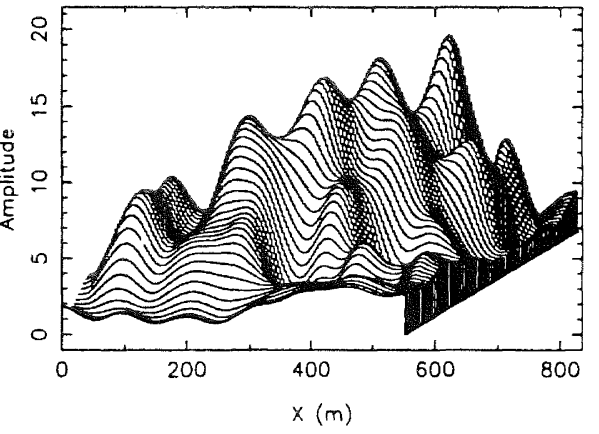

Level 0 of Alfredton Basin

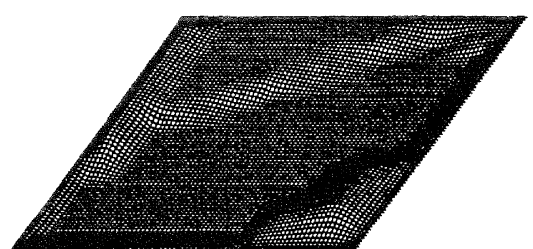

FIGURE 15. Amplitude distributions at the ground surface of the fundamental wavefields responding to the same plane incident $\mathrm{SH}$ waves of $3 \mathrm{~Hz}$ as in Figure 11 (bottom), where the solution in the basin has been obtained with 69 horizontal wavenumbers. Here 109 (top) and 145 (bottom) horizontal wavenumbers have been used instead. 


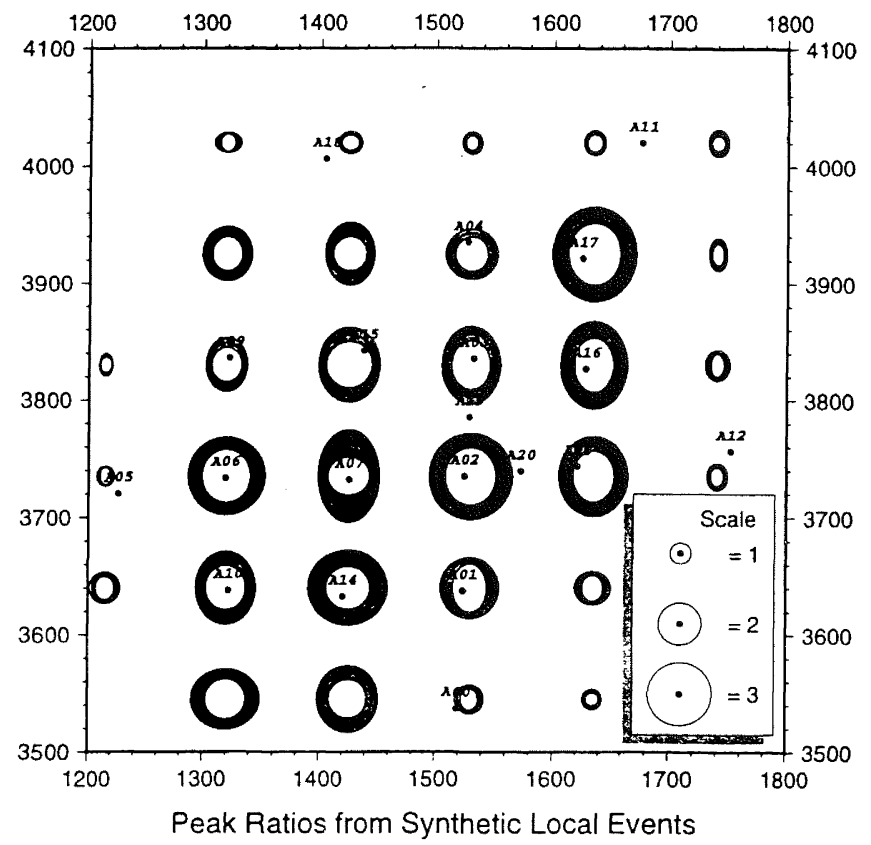

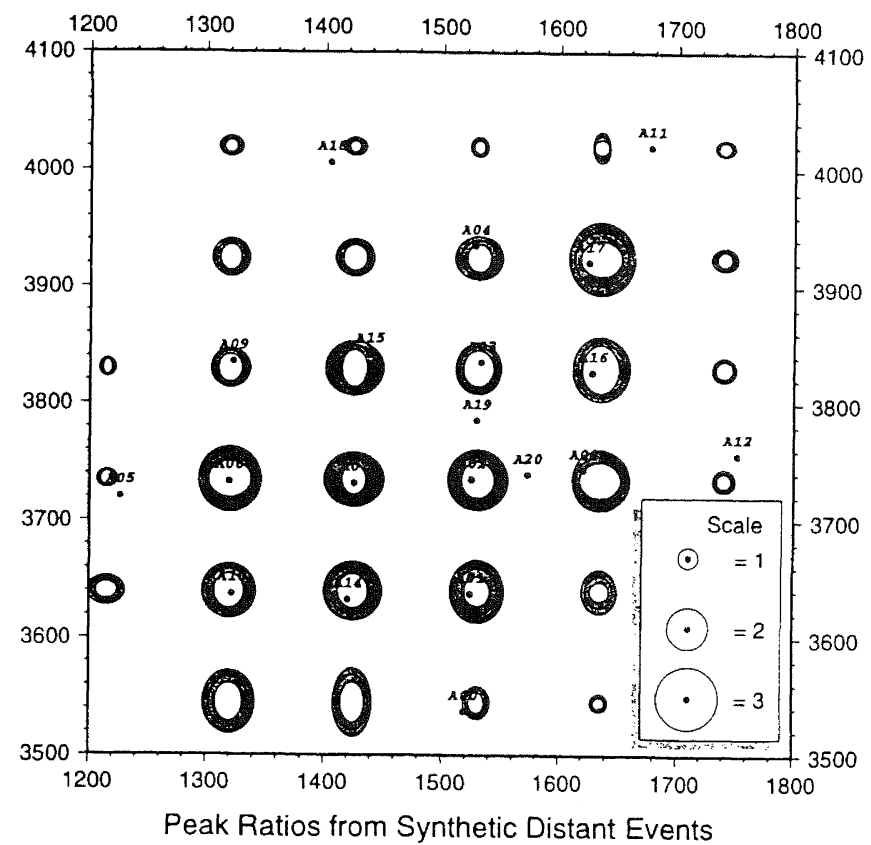

FIGURE 16. Statistics of the amplification of east-west and north-south horizontal peak ground displacement, for (a) synthetic events resembling the nearby event 9 and (b) synthetic events resembling the more distant event 12. The means and standard deviations are presented in the same way as in Figure 7, with the values for the individual synthetic events having been normalised with respect to the RMS values from firm sites near the reference sites used for the observations.

spatially incoherent as those for the weak-motion events we have observed and modelled. In other words, we have no data on what the coherency properties of the incident wavefields will be like at Alfredton for a large earthquake. On the top of this is the question of nonlinear behaviour of the sediments in the basin in earthquakes that are large enough. Another area of uncertainty is whether the amplifications for shallow earthquakes which excite significant surface waves, will be larger than from the lower crustal and deeper subduction-zone events that were observed. In this case, we can infer from the modelling that larger amplifications from shallow earthquakes are unlikely at Alfredton, as is illustrated by the low amplifications for surface waves in Figure 14.

Both the observations and the modelling show that the amplifications of the peak motions and the Fourier spectra of the motions in the basin at Alfredton are smaller than in situations where there are large abrupt changes in shear-wave impedance to trap seismic energy in the low-velocity soft-soil layers. In particular, because of the lack of any such boundary to trap the energy, the duration of the ground motion is not noticably longer in the basin than at the surrounding firm sites. The lesson from this is that the presence of an appreciable thickness of soft soil is not necessarily indicative of a major seismic hazard comparable to that in Mexico City, unless there are large contrasts in shear-wave impedance at the boundaries between soil layers or between the soft soil and the underlying basement rock.

\section{ACKNOWLEDGEMENTS}

The work in this paper has been part of the Seismic Hazard Microzoning Programme at IGNS, to which Jiashun Yu in the course of his Ph.D. studies has been a major contributor. John
Haines thanks FRST for support of his part of the joint effort which has consisted primarily of the computer coding for the basic modelling and for the generation of the synthetics with randomly chosen weights to simulate spatially incoherent incident wavefields, and both John and Jiashun thank EQC for funding the first three years of Jiashun's Ph.D. and IGNS for subsequent support. We thank also John Taber and John Zhao for their comments on the paper. As well, John Taber has with John Haines in their joint supervision of Jiashun contributed towards the development of Jiashun's skill in interpreting the observed and synthetic data.

This paper has been written because of the need to publicise the modelling results, which would have been meaningless without presenting sufficient of the observational data to place the modelling into context. John Haines is especially grateful to Jiashun for allowing him to do so at the same time as Jiashun is completing his thesis. The data that are presented here have been selected to emphasise the incoherent nature of the incident wavefields and to give an indication of the character of the observed amplifications, and the interpretations are focussed on explaining why the modelling took the form that it did. The presentation by Jiashun in his thesis is a complete analysis, which he will be presenting in subsequent papers.

\section{REFERENCES}

Aki, K. and P.G. Richards, 1980. Quantitative seismology, (2 volumes), W.H. Freeman and Company, San Francisco.

Barker, P.R., 1994. A report on cone penetrometer and seismic cone penetrometer testing at selected sites in lower North 
Island, Consultancy report for Institute of Geological \& Nuclear Sciences.

Begg, J.G., D.C. Mildenhall, G.L. Lyon, W.R. Stephenson, R.H. Funnell, R.J. Van Dissen, S. Bannister, L.J. Brown, B. Pillans, M.A. Harper and J. Whitton, 1993. A palaeoenvironmental study of subsurface Quaternary sediments at Wainuiomata, Wellington, New Zealand, and tectonic implications, New Zealand Journal of Geology and Geophysics, 36:461-473.

Benites, R.A. and A.J.Haines, 1991. The Riccati Matrix Equation-Boundary Integral method to compute seismic wavefields in heterogeneous media, EOS. Transactions of the American Geophysical Union, 72(44):332 (abstract).

Borcherdt, R.D. and G. Glassmoyer, 1992. On the characteristics of local geology and their influence on ground motions generated by the Loma Prieta earthquake in the San Francisco Bay region, California, Bulletin of the Seismological Society of America, 82:603-641.

Davis, R., 1995. Effects of weathering on site response, Earthquake Engineering and Structural Dynamics, 24:301-309.

Gutierrez, G. and S.K. Singh, 1992. A site effect study in Acapulco, Guerrero, Mexico: a comparison of results from strong-motion and microtremor data, Bulletin of the Seismological Society of America, 82:642-659.

Haines, A.J., 1989. On the structure of one-way elastic-wave propagation and exact solutions to the elastodynamic equations: theory for 2-dimensional SH waves, Geophysics Division, DSIR, 259pp. (Reissued, Institute of Geological \& Nuclear Sciences, Science Report 95/8, 1995).

Haines, A.J. and M.V. de Hoop, 1996. An invariant imbedding analysis of general wave scattering problems, Journal of Mathematical Physics, 37:3854-3881.

Haines, A.J., W.R. Stephenson and J. Yu, 1994. The Alfredton soft-soil-site basin-response experiment: peak velocities, Proceedings of the Technical Conference of the New Zealand National Society for Earthquake Engineering, 200-205.
Stephenson, W.R., 1993. The Alfredton soft soil site, Institute of Geological \& Nuclear Sciences, Science Report.

Stephenson, W.R. and P.R. Barker, 1992. Evaluation of sediment properties in the Lower Hutt and Porirua areas by means of cone and seismic cone penetration tests, Bulletin of the New Zealand National Society for Earthquake Engineering, 25:265-285.

Taber, J.J., 1993. Instrumental recording of microzonation effects, Proceedings of the Technical Conference of the New Zealand National Society for Earthquake Engineering, 119-124.

Taber, J.J. and H.A. Cowan, 1993. Measurement of earthquake ground shaking at characteristic sites in Christchurch, Report to the Earthquake and War Damages Commission and the Canterbury Regional Council.

Taber, J.J. and E.G.C. Smith, 1992. Frequency dependent amplification of weak ground motions in Porirua and Lower Hutt, New Zealand, Bulletin of the New Zealand National Society for Earthquake Engineering, 25:303-331.

Wu, R.S. and K. Aki, 1985. Scattering of elastic waves by a random medium and the small scale inhomogeneities in the lithosphere, Journal of Geophysical Research, 90:10261-10276.

Yu, J., 1996. Observation and synthesis of seismic wavefields in basin structures, Ph.D. thesis, Victoria University of Wellington, (in prep.).

Yu, J. and A.J. Haines, 1994. Observation and synthesis of seismic wavefields in basin structures - analysis of seismic wave amplification at Alfredton basin, New Zealand, Report to the Earthquake Commission.

Zhao, J.X., D.J. Dowrick and G.H. McVerry, 1997. Attenuation of peak ground accelerations in New Zealand earthquakes, Bulletin of the New Zealand National Society for Earthquake Engineering, (in prep.). 